\title{
Common infectious diseases of the central nervous system - clinical features and imaging characteristics
}

\author{
Shan Li^, Ivy P. Nguyen, Kyle Urbanczyk \\ Department of Radiology, Baystate Medical Center, University of Massachusetts School of Medicine-Baystate, Springfield, MA, USA \\ Correspondence to: Shan Li, MD, MS. Assistant Professor, Department of Radiology, Baystate Medical Center, University of Massachusetts School of \\ Medicine-Baystate, 759 Chestnut Street, Springfield, MA 01199, USA. Email: Shan.Li@baystatehealth.org.
}

Submitted Jun 16, 2020. Accepted for publication Aug 03, 2020.

doi: 10.21037/qims-20-886

View this article at: http://dx.doi.org/10.21037/qims-20-886

\section{Introduction}

The central nervous system (CNS) primarily consists of the brain and spinal cord, which are enclosed within the surrounding meninges and bone structures such as the calvarium and spine. Infectious diseases of the CNS include a wide spectrum of infections caused by various pathogens affecting one or more of these components as well as the spaces between these components. Different components or compartments of the CNS are affected circumstantially; each of the disease entities will have different imaging pattern and characteristics according to their different transmission routes, locations and appearance, i.e. meningitis, brain abscess, septic emboli, spinal epidural empyema and osteomyelitis (OM). Despite the advances of modern medicine, accurate diagnoses of a specific disease entity of the CNS infections have proven to be very challenging as their clinical presentations are very general and nonspecific, such as fever, headaches, weakness, numbness or back pain.

The infectious diseases of the CNS are most commonly caused by various types of bacteria or viruses. Other less common organisms include fungi or protozoans, etc. These pathogens enter the CNS through two main routes of transmission: hematogenous spread from distant infections such as endocarditis or urinary tract infection, or direct extension from infections of the adjacent structures such as sinusitis or mastoiditis. Hematogenous transmission tends to produce diffuse or multifocal lesions in different vascular distributions, such as diffuse meningitis or septic emboli. Direct extension of the infections usually gives rise to focal lesions, such as subdural or epidural empyema. Whenever local infection is identified, the adjacent anatomic structures (e.g., paranasal sinuses, mastoid air cells, middle ears) should be examined diligently to pinpoint the initial source. Extended involvement of other CNS compartments and vascular systems is not uncommon, as it can coexist or become complications of primary meningitis or encephalitis, such as ventriculitis or thrombophlebitis.

Neuroimaging techniques are essential for diagnosing various CNS infections and help differentiate different disease entities. The two main utilized imaging modalities are computed tomography (CT) and magnetic resonance imaging (MRI), each with their own advantages and limitations. CT scanners are widely available in hospitals and clinics, being operated 24 hours a day. Nonenhanced CT (NECT) images can be acquired quickly and with little patient preparations. CT scan is highly sensitive to acute intracranial hemorrhages (parenchymal or extraaxial), calcified lesions or bony deformities. NECT of head often serves as a gateway for more advanced imaging or more complexed patients' management, such as screening for lumbar puncture (LP) or used for CT-guide biopsy or drainage. Administration of intravenous contrast in CT usually increases the sensitivity of detecting an underlying abnormality and further delineating its characters. Vascular

\footnotetext{
^ ORCID: 0000-0002-9517-4278.
} 
complications of CNS infections can be accessed by CT angiogram of head, either in arterial or venous structures. The main drawback of CT is its radiation to patients. Other disadvantages include relatively low soft tissue resolution, limited imaging display planes and less sensitive contrast enhancement. Small extra-axial fluid collections can be missed. Meningitis or other subtle structural changes may not be detected at early stages. MR imaging is much more sensitive for detecting early changes of CNS infections and depicting various imaging findings during each disease process, due to its high anatomy resolution, superb soft tissue differentiation, multiplanar acquisition, and versatile sequences of delineating different characters of pathologic processes of CNS infections. Vasogenic edema caused by infection appears hyperintense on T2-weighted (T2WI) or FLAIR images; A small hemorrhagic focus within the infection could may manifest as an exaggerated blooming artifact on gradient echo sequence (GRE); Hyperintense signals on diffusion weighted images (DWI) are highly characteristic of many types of infectious lesions and separating them apart from other etiologies. Administration of intravenous contrast in MRI increase the sensitivity and specificity of the MRI technique, helping further delineate the pathology, exclude most noninfectious etiologies, narrow down the differential diagnoses. The disadvantages of MR include lesser available MR scanners in hospitals, longer examination time and certain limitations of the patients. Claustrophobia could be a problem for some patients. Patients with pacemakers or other batteryoperated devices as well as metallic foreign bodies in critical organs are also contraindications for MR, although MRcompatible pacemaker started to appear on the market in recent years.

Additionally, vascular complications of CNS infections, either in arterial or venous compositions can be accessed by CT or MR angiogram/venogram, although DSA remains the gold standard for evaluating these conditions.

Side effects of contrast such as allergic reactions can be seen using either iodinated contrast for CT or DSA and Gadolinium containing contrast for MR. Both contrasts should be used with caution for patients with renal failure. Nephrogenic systemic fibrosis is a special disease entity related to the Gadolinium composition of MR contrast.

In this article, we have reviewed various infectious diseases of the CNS, described their clinical presentations, laboratory findings, imaging characteristics and patterns, differential diagnosis and treatment options. Most importantly, a comprehensive review of the patient's clinical picture prior to reviewing imaging can help direct the search and identify subtle but expected abnormalities that might be otherwise overlooked.

\section{Acute meningitis/encephalitis}

Acute meningitis is an infection of the membranes (meninges) covering the brain and spinal cord. It is the most common infectious disease of the CNS. Either leptomeninges (pia and arachnoid matter) or pachymeninges (dura matter) can be affected. The leptomeningeal meningitis is more common than the pachymeningeal meningitis and is more conspicuous on neuroimaging.

The most common etiology of acute meningitis is bacteria. Other common pathogens include viruses and fungi. Protozoans or parasites are less commonly seen in Western countries. The bacterial cases are usually more fulminating than viral ones and often life-threatening but not without exceptions. In newborns, group B Streptococcus, gram-negative bacteria such as E. coli, Klebsiella-Entero and pseudomonas are the main causes of meningitis. Other pathogens include staphylococci and listeria monocytogenes. Beyond the newborn period, the most common causes of bacterial meningitis are Neisseria meningitidis, Streptococcus pneumoniae, and Hemophilus influenza which account for $80 \%$ of cases (1-6). All three of these organisms are respiratory pathogens. They first colonized within the mucosa of nasopharynx, oropharynx or larynx, then entered the bloodstream. The most common route of transmission is through hematogenous spread. Others gain access to the CNS via direct spread from local infectious foci, after head trauma or neurosurgery, or during vaginal delivery from untreated GBS-colonized mothers.

The classic triad of clinical presentations of bacterial meningitis consists of fever, headache and nuchal rigidity, although most patients rarely present with all three of the textbook definitions. They often have at least two of the four symptoms of headache, fever, neck stiffness, and altered mental status. Other common presentations include seizures, and focal neurologic deficits. CNS symptoms depend on areas of involvement, cerebral, cranial nerve or spinal nerves. In infants and young children, decreased oral intake, temperature instability, lethargy, and seizures are vague clinical clues calling for a higher index of suspicion for more serious diseases (4-6).

Diagnosis of meningitis is mainly based on clinical findings in suspected patients combining typical symptoms and abnormal lab tests, such as positive CSF gram stain 
or culture. LP remains the gold standard for diagnosing meningitis. A head CT should be performed before LP to exclude increased intracranial pressure due a mass or other contraindication. Positive cerebral spinal fluid (CSF) cytology is found on the initial LP in $50-70 \%$ and in nearly all cases after 3 attempts. Characteristic CSF findings include increased $\mathrm{WBC}$ count $>1,000 / \mathrm{mL}$; elevated protein level $>200 \mathrm{mg} / \mathrm{dL}$ and decreased glucose $<40 \mathrm{mg} / \mathrm{dL}$. The reported sensitivity of Gram stain for bacterial meningitis has varied from $50 \%$ to $90 \%$; however, the specificity approaches $100 \%$ when present. CSF culture yield is low and can be negative when blood culture is positive. But blood culture does not point to the source and location of the infection. The yield of Gram stain and culture are significantly reduced if patients have received antibiotics before LP. Other CSF tests such as polymerase chain reaction (PCR) or Latex agglutination test (LAT) are not routinely recommended. However, LP is a labor-intensive interventional procedure, requiring trained medical personnel. Other major drawbacks include the laboratory time required for CSF culture $(7,8)$.

Neuroimaging is extremely valuable in evaluation for meningitis. Nonenhanced CT (NECT) of brain may be normal in early state of meningitis, although negative result cannot exclude the presence of meningitis. Mild sulci effacement, mild ventriculomegaly or acute obstructive hydrocephalus could be an early indication of meningitis. Once the exudate from the inflamed meninges replaces CSF, the previously clear CSF may become almost isodense to brain parenchyma on NECT. Following IV contrast administration, the inflamed meninges and sulci become intensely enhanced. Gadolinium-enhanced MRI is the preferred imaging modality over CT because of its sensitivity and specificity. On MRI, the purulent exudates are $\mathrm{T} 1$ isointense with brain parenchyma and $\mathrm{T} 2$ isointense with CSF, but hyperintense in subarachnoid spaces on fluidattenuation inversion recovery (FLAIR) MRI (9). The meninges and subarachnoid spaces often show restricted diffusion on diffusion weighted imaging (DWI). Enhanced MRI shows either diffuse "sugar coating" or thicker, lumpy, multinodular enhancement along the affected letpo- or pachy-meninges. Up to $50 \%$ of cases exhibit subarachnoid enhancement with curvilinear patterns that highlight the gyri and sulci. Dura-arachnoid space enhancement is less frequently seen. Post-contrast FLAIR increases the sensitivity of detecting subtle cases (10-12) (Figure 1).

In addition to meningitis, the underlying brain parenchyma often shows coexisting encephalitis or can become affected as a complication of meningitis. The encephalitis is in close proximity of the meningitis with the involved brain parenchyma show similar hypointense T1 and hyperintense T2/FLAIR signals as those of meningitis. It also shows significant restricted diffusion which does not follow the vascular distribution. This feature helps its differentiation from a common infarct as a result of vessel occlusion due to thrombus or atherosclerosis. Its enhancement pattern is variable and ill-defined, unlike the mass-like appearance of a neoplasm. Other complications of meningitis include brain abscess, subdural/epidural empyema, ventriculitis, venous thrombosis, obstructive hydrocephalus, etc. They often demonstrate markedly different imaging findings, some of which will be discussed later in this article.

Differential diagnoses of meningeal enhancement include meningeal carcinomatosis, intracranial hypotension, reactive inflammation after LP, etc. Patients' clinical history and presentations often provide diagnostic directions. The sulcal-cisternal FLAIR hyperintensity is not specific and can present in other conditions like subarachnoid hemorrhage, CSF metastases, susceptibility or flow artifact, or simply due to a patient being on $100 \%$ oxygen support (12).

Prompt and high-dose antibiotics are the mainstay of treatment, with the best being organism-specific. Broad spectrum antibiotics are used in case there is no known causative agent. The use of dexamethasone to reduce intracranial inflammation is advocated after numerous studies proving its association with decreased mortality in bacterial meningitis. Survivors of meningitis/encephalitis often suffer long-term neurological deficits, such as hearing loss, cognitive or motor deficit, visual impairment.

\section{Herpes simplex encephalitis}

There are two types of Herpes simplex viruses (HSV), Herpes simplex virus type I (HSV-1) and Herpes simplex virus type II $(H S V-2) . H S V$ infections in human are extremely common, with almost half of the US population aged 14-49 seropositive for $H S V-1$ and $11.9 \%$ seropositive for HSV-2 (13).

$H S V-1$ is oral herpes, transmitted by oral to oral contact. It is often acquired silently earlier in life, staying dormant within the body and becoming reactivated during adulthood.

$H S V-1$ encephalitis is the most common cause of sporadic fatal encephalitis worldwide, accounting for $10-20 \%$ of the roughly 20,000 annual viral encephalitis 

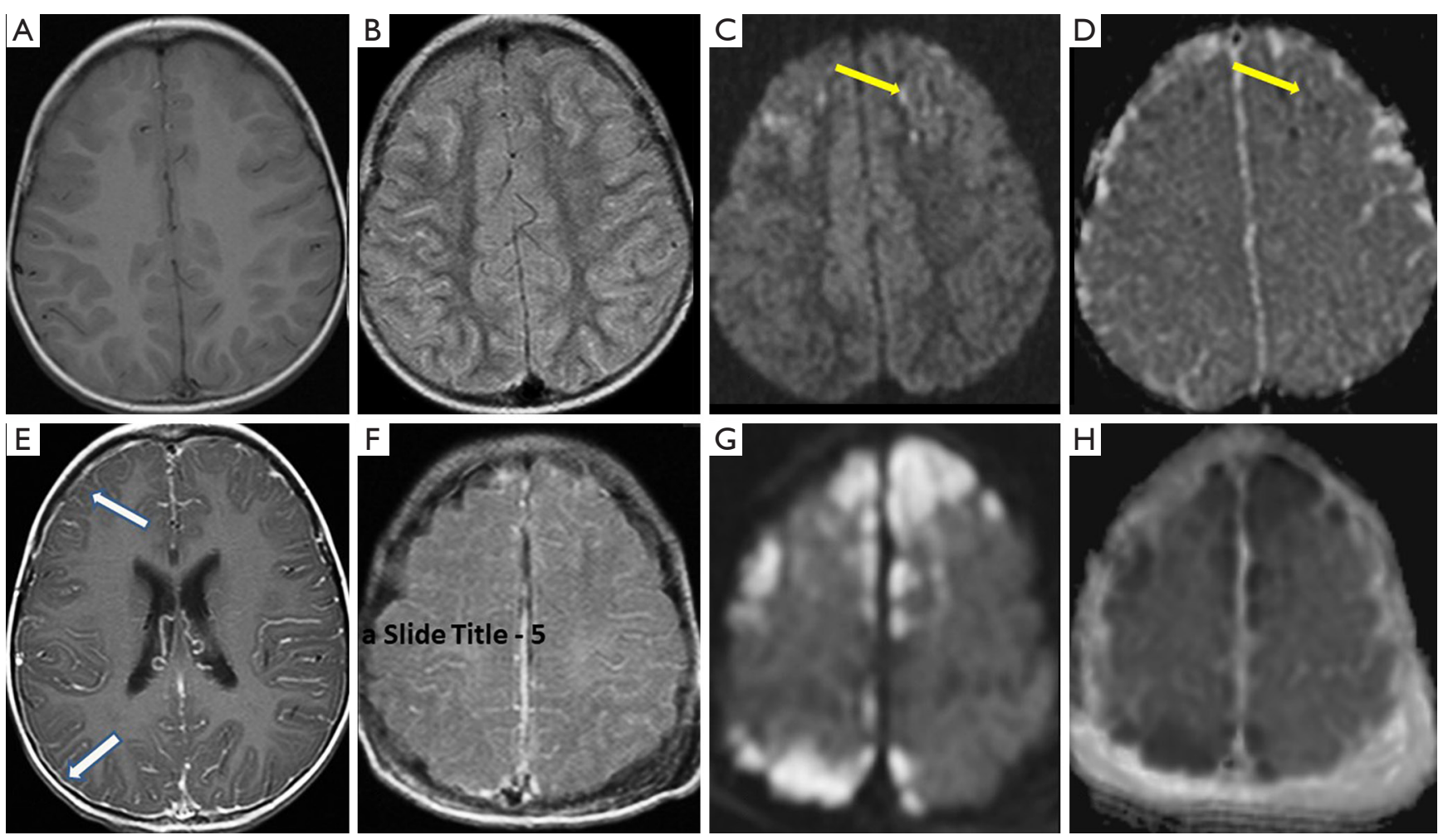

Figure 1 Acute meningitis. A 2-year-old boy presented with high fever of 103 (F), neck stiffness, and right eye ptosis after 4 days of runny nose, vomiting, diarrhea and decreased oral uptake. NECT was normal (not shown). On MR, axial T1 image (A) and T2 image (not shown) showed no abnormal signal. On axial FLAIR image (B) there were diffusely increased signals within the sulci of bilateral cerebral hemispheres. A few focal areas of restricted diffusion were seen within the sulci of bilateral frontal regions (yellow arrow) on axial DWI and ADC images (C,D), suggesting purulent materials. Post contrast axial T1 image (E) showed diffuse enhancement along the leptomeninges of bilateral cerebral hemispheres consistent with leptomeningitis. Subtle dural enhancement was also seen at multiple locations (blue arrow), consistent with pachymeningitis. CSF culture grew Streptococcal Pneumoniae. In another patient with diffuse meningitis on post contrast axial FLAIR image (F), multiple areas of restricted diffusion were noted in the underlying brain parenchyma on axial DWI and ADC images $(\mathrm{G}, \mathrm{H})$, suggesting associated encephalitis or acute ischemia. (All images of these cases are from the Hospitals of the University of Massachusetts School of Medicine at Baystate Health System.) NECT, nonenhanced CT; FLAIR, fluid-attenuation inversion recovery; DWI, diffusion weighted images; ADC, apparent diffusion coefficient; CSF, cerebral spinal fluid.

cases. All age groups are affected, with a third of all cases occurring in children and adolescents. $H S V-1$ encephalitis includes nearly all cases of $H S V$ encephalitis after early infancy. It is caused by reactivation of the dormant $H S V$ 1 virus. $H S V-1$ can be harbored in the human body in a dormant form indefinitely, usually in the Gasserian ganglia of the trigeminal nerves. The reactivation of the virus enters the brain through the trigeminal nerve pathway to preferentially affect the ipsilateral temporal lobe. Bilateral temporal lobe involvement is less common but usually asymmetrical $(14,15)$.

$H S V-1$ encephalitis is fulminant necrotizing encephalitis.
The patients' clinical presentations are usually abrupt but nonspecific, consisting of sudden onset of fever, headache, seizures, and impaired consciousness, etc. The symptoms often progress with rapid deterioration, with a mortality rate of $70 \%(13,14)$. CSF analysis is nonspecific and commonly shows a lymphocytic pleocytosis, increase erythrocytes (RBC) and elevated protein. Definitive diagnosis is made by detecting $H S V-1$ DNA in CSF by PCR testing which has a very high sensitivity and specificity, but the result often takes days to reveal (14). Due to its invasive nature, brain biopsy is not usually required unless in ambivalent or difficult cases. Early intervention by intravenous acyclovir 

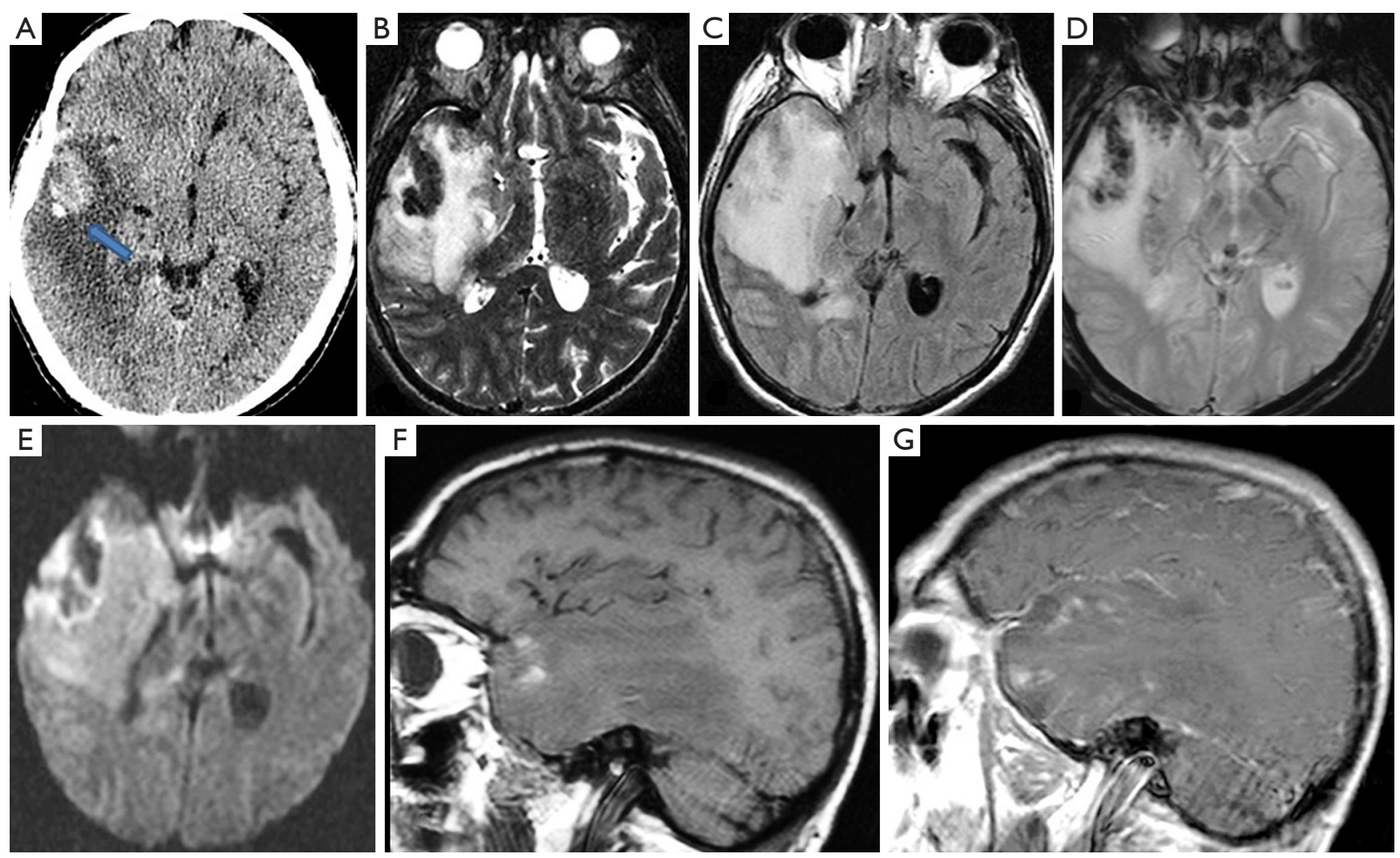

Figure 2 Herpes simplex encephalitis type I. A 52-year-old male was found confused, incontinence with garbled speech. He was febrile of 101.9 F. Axial NECT (A) showed a large area of hypodensity in the right temporal lobe with significant swelling and mass effect. A small hyperdense focus within the lesion was consistent with acute hemorrhage (arrow). Acute subarachnoid hemorrhages were also seen in the adjacent right Sylvian fissure. On MR, the right temporal lobe contained a large area of heterogeneously hyperintense signals on axial T2 and FLAIR images (B,C). It also appeared hyperintense on axial DWI (E) without corresponding hypointense ADC signal (not shown). The findings could a combination of infection, edema and ischemia. The hypointense foci within the lesion were consistent with hemorrhages, which appeared more conspicuous on axial GRE image (D) and DWI (E). On pre- and post-contrast axial T1 (F,G) image, the right temporal lobe lesion demonstrated multiple enhancing foci as well as leptomeningeal or vascular enhancement within the related sulci. The patient's PCR of CSF was positive for HSV-1. (All images of this case are from the Hospitals of the University of Massachusetts School of Medicine at Baystate Health System.) NECT, nonenhanced CT; DWI, diffusion weighted images; ADC, apparent diffusion coefficient; FLAIR, fluid-attenuation inversion recovery; GRE, gradient echo sequence; PCR, polymerase chain reaction; CSF, cerebral spinal fluid.

could be lifesaving when $H S V$ encephalitis is suspected but not confirmed.

Temporal lobe abnormalities on neuroimaging are highly suspicious of $H S V-1$ encephalitis, although in early stages, CT of brain with and without contrast could be normal. Suspicion for herpes encephalitis should never be excluded based on a normal CT. Half of the patients usually demonstrate abnormalities on NECT, with low density noted in the temporal lobe and insular cortex, along with mild mass effect. In advanced stages, the affected areas show significant swelling, focal hemorrhages, necrosis and heterogeneous enhancement. On MRI, FLAIR images show asymmetric hyperintensity of the temporal lobes and insula with some restricted diffusion on DWI, correlating histologically with cytotoxic edema. In one study, DWI was found to be slightly superior in diagnosing acute and subacute herpes encephalitis $(15,16)$. Susceptibility weighted imaging (SWI) are more sensitive to show petechial hemorrhages in early disease course which can be easily overlooked on NECT. Contrast enhanced images may show leptomeningeal enhancement and variable gyriform or parenchymal enhancement. Symmetrical involvement of 
both temporal lobes and the lateral putamen is atypical and may be suggestive of alternate disease processes (Figure 2).

Differential diagnosis include other types of bacterial or viral encephalitis as their symptoms and imaging findings can sometimes be the same and definitive diagnosis can only be made by brain biopsy. Other differential diagnoses include limbic encephalitis, glioma, infarct, etc. Clinical presentations of these conditions are significantly different from $H S V-1$ encephalitis and much less fulminant. Limbic encephalitis is a group of autoimmune diseases involving the limbic system, including medial temporal lobe, hippocampus, amygdala, etc. It often has a subacute onset of symptoms, such as short-term memory loss, seizures and psychiatric symptoms, and can be associated with cancer within 5 years of diagnosis. Key imaging features which help to differentiate herpes encephalitis from limbic encephalitis is that herpes encephalitis will almost always spare the basal ganglia and is usually unilateral involvement, whereas limbic encephalitis usually involves the basal ganglia and often is bilateral. On MR, limbic encephalitis commonly shows hyperintense T2 signals without hemorrhage or enhancement. A glioma also has an insidious onset. A low grade one shows an infiltrative lesion with hyperintense T2 signals and mild mass effect, but no associated hemorrhage and does not enhance. A higher-grade glioma becomes more heterogenous with significant vasogenic edema and mass effect. It shows areas of necrosis and hemorrhages as well as heterogenous enhancement. Restricted diffusion is usually absent. An infarct of the temporal lobe follows the vascular territories, i.e., an MCA infarct involves the anterior and superior temporal lobe by MCA and a PCA infarct involves the inferior temporal lobe. Its signal intensities on DWI are more intense and more uniform than that of the encephalitis.

$H S V-2$ is genital herpes, characteristically affecting neonates through direct transplacental transmission or by perinatal transmission via the birth canal. The incidence is about 3-30 per 100,000 live births. In the United States, there are an estimate 1,500 cases of neonatal HSV2 infection annually (17). Neonate $H S V-2$ infections can manifest as three forms: localized type with skin, eye and mouth involvement (SEM), CNS infection (encephalitis) without or with SEM, disseminated type. In this article, we concentrate on neonatal $H S V$-2 encephalitis.

$H S V-2$ encephalitis involved in approximately $30 \%$ of $H S V-2$ infected neonates. The infected neonates tend to present at 2-4 weeks after birth with fever, seizures, irritability, lethargy and poor feeding. The symptoms can deteriorate rapidly to coma and death. CSF analysis usually shows a mononuclear cell pleocytosis, normal or moderately low glucose and mildly elevated protein. Elevated RBC count is not usually present. Like HSV1, the standard diagnostic test for $H S V-2$ is also the CSF PCR to detect $H S V-2$ DNA in CSF $(75-100 \%$ positive) $(16,17)$.

Prenatal ultrasound of head or fetal MRI may show brain damages or congenital anomalies but results are nonspecific, and the yield is low. Neonatal ultrasound often underestimates the extent of brain damages and should not be used as the sole imaging study in suspected cases (16). NECT of head could be normal at the beginning but quickly show multiple areas of hypo-attenuation, loss of gray-white matter differentiation and sulci effacement in the brain, consistent with brain destructions and vasogenic edema. MRI is more sensitive and may show an early loss of the gray-white distinction with heterogeneously T2 hyperintensity and variable restricted diffusion in the cortex and white matters. Post contrast images show various meningeal or parenchymal enhancements of the diseased areas. Unlike $H S V-1, H S V-2$ is nonspecific in its regional involvement and may be diffuse or localized but does not show preference for any particular area of the brain parenchyma. In late stages, the affected brain shows multiple cystic encephalomalacia, volume loss and dystrophic calcifications $(13,18)$ (Figure 3).

Differential diagnoses include acute meningoencephalitis of either bacterial or viral types, or acute disseminated encephalomyelitis (ADEM). Findings of acute meningoencephalitis have been discussed in the above section above. ADEM usually occurs in children between 3-7 years old and often has a prodrome of viral infection (75\%). Neuroimaging studies typically demonstrate diffuse, poorly demarcated, large lesions predominately in cerebral white matters. Bilateral basal ganglia are often involved as well.

Despite treatment, prognosis of HSV-2 encephalitis remains poor and the mortality rate is high. Survivors often have long-lasting neurological damages including seizure disorders, mental or motor retardation, microcephaly, etc.

\section{Eastern equine encephalitis (EEE)}

Acute encephalitis caused by arboviruses in the United State is very rare but nonetheless it often captures the headlines of the national or regional news when it happens due to the devastating nature of the disease. Five types of arboviruses found in the US can cause encephalitis: Eastern equine 

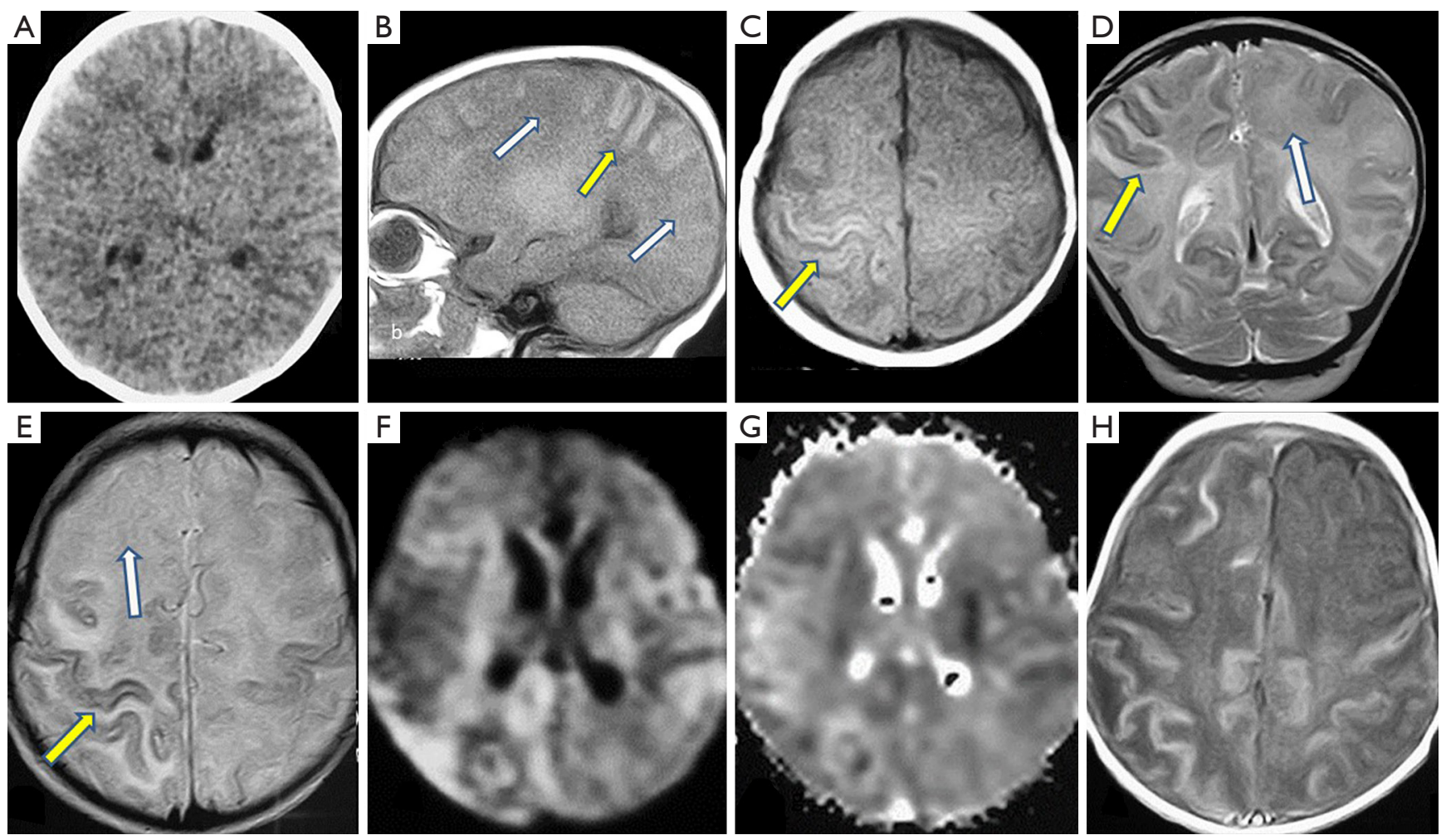

Figure 3 Herpes simplexencephalitis Type II. A 25-day-old baby presented with fever, respiratory distress and decreased oral uptake. Axial NECT (A) was unremarkable without evidence of cortical infarct or hemorrhage. On MR, sag and axial T1 images (B,C) showed multiple areas of hypointense signals within the cerebral hemispheres with loss of gray-white matter differentiation (blue arrow). These areas appeared isoor hypointense on coronal T2 (D) image. There were also some gyriform hyperintense T1 along the cortex (yellow arrow) which appeared hypointense on axial T2 and GRE images (D,E). They might represent petechial hemorrhages or dystrophic calcifications. Bilateral cerebral hemispheres demonstrated various areas of restricted diffusion on axial DWI and ADC images (F,G) suggesting acute brain injury. Post contrast axial T1 image (H) showed multiple areas of cortical and leptomeningeal enhancement of the cerebral hemispheres with diffuse effacement of the sulci and areas of indistinct corticomedullary junction. The findings were consistent with meningoencephalitis. The patient's CSF study was positive for HSV-2. (All images of this case are from the Hospitals of the University of Massachusetts School of Medicine at Baystate Health System.) NECT, nonenhanced CT; DWI, diffusion weighted images; ADC, apparent diffusion coefficient; CSF, cerebral spinal fluid.

virus, Western equine virus, West Nile virus, St. Louis virus and La Crosse virus. They account for $10 \%$ of cases of encephalitis. EEE is the most severe and fulminating type of encephalitis among those. It has a mortality rate of at least $30 \%$, the highest among all arbovirus-caused encephalitis. From 2003 to 2016, a total of 121 human cases were reported, with a median of 8 cases reported annually. The national average annual incidence was 0.03 cases/ million people (19). The annual cases rose dramatically in 2019 during which, 38 new cases were reported including 15 deaths.

Eastern equine virus is a mosquito-borne virus, found in the northeast regions of the US, the Great Lakes and along the Gulf Coast. Originally this virus was identified from the horses in Massachusetts in 1831. It was later also identified in other animals such birds and reptiles. Humans acquire EEE through the bite of an infected mosquito releasing the viruses into the bloodstream. The infected mosquito in turn acquires the virus through biting the infected animals and sucking their blood. The first identified human case was in 1938 when there was an outbreak in Massachusetts during which there were 25 deaths from 34 cases (20).

In the United States, although EEE can be sporadic occurring throughout the year, small outbreaks tend to occur each summer. Most people bitten by infected mosquitos are asymptomatic or show mild symptoms such 
as low-grade fever, malaise, and joint or muscle cramps. Less than $5 \%$ of infected people develop acute encephalitis. Patients over 50 years old or younger than 15 years old are at higher risk. Patients on immunosuppressant therapy are also more vulnerable.

Patients usually present with nonspecific but rapidly progressing symptoms at about 4-10 days after the infected mosquito bite: sudden onset of high fever, chills, headaches, vomiting and seizures. Any travel history or outdoor activities should be carefully examined. The symptoms deteriorate quickly to disorientation, coma, and death. Survivors generally have severe sequelae including convulsions, paralysis, and mental retardation.

Diagnosis of EEE is difficult as the clinical picture is often vague. Diagnosis is usually made by demonstrations of immunoglobulin $\mathrm{M}$ (IgM) antibody to EEE in CSF or a 4 time increase in serum antibody titers against EEE virus. Neutrophilic pleocytosis may be seen in CSF which is unusual in viral encephalitis (21). In some cases, virus, viral antigen or viral genomic sequences can be isolated from tissue, blood, or CSF. Serum IgM antibodies alone should be confirmed by demonstration of virus-specific neutralizing antibodies. The specimens must be sent to special laboratories in the country and the results are not available until a few days to a couple of weeks after specimen receipt.

Neuroimaging findings are very helpful as they often become abnormal in early stage. On NECT, diffuse heterogeneous hypointense areas are seen involving bilateral basal ganglia and thalami, associated with significant swelling and mass effect. The lateral ventricles and the third ventricle are effaced. Usually there is no evidence of frank hemorrhages. On MRI, heterogeneously hypointense T1 and hyperintense T2/FLAIR signals within bilateral basal ganglia and thalami appear to be striking features and persistent in multiple reports. This is thought to be due to combination of edema, inflammation and ischemia $(21,22)$. Particularly linear areas of hyperintense T2/FLAIR signals within the external and internal capsules with relative sparing of the lentiform nuclei are seen in considerable amount of cases for which a "parentheses sign" has been proposed which may represent a distinguishable feature of EEE (23). The validity of this "parentheses sign" is still debatable and further confirmation is needed when more imaged cases are available. In some cases, abnormal signals extend to the midbrain and pons. DWI shows isointense signals without obvious restricted diffusion. In most cases, no enhancement is noted. There are also some reports exhibiting focal areas of hyperintense T2/FLAIR signals affecting the cortex and subcortical white matters of cerebral hemispheres (Figure 4).

Other types of arthropod-borne viral encephalitis such as West Nile encephalitis, St. Louis encephalitis, show similar imaging findings and should be included in the differential diagnoses. Other diseases involving bilateral basal ganglia and thalami include Creutzfeldt-Jacob disease and hypoxicanoxic encephalopathy, which have distinct clinical histories, presentations and lab tests. Persistent hyperintense diffusion signals of the diseased areas are most sensitive signs and differentiate them from EEE.

There is no effective anti-viral drug for EEE. Treatment is mostly supportive, including intravenous fluids, intubation, steroids and anticonvulsant. One thirds of patients die despite treatment. Fifty percent of survivors suffer permanent brain damage. At present, no human EEE vaccine is available.

\section{CNS tuberculosis}

Tuberculosis infection remains a leading cause of morbidity and mortality in developing countries, which is caused by Mycobacterium tuberculosis (TB), a contagious acid-fast bacillus. According to World Health Organization (WHO), the global incidence of TB is estimated at 10 million, with approximately 1.5 million patients died of TB in 2018 (24). In the United States, the incidence of TB infection is lower; most cases $(70 \%)$ occur in immigrants from countries with high rates of endemic TB, according to the Center of Disease Control and Prevention (CDC) $(25,26)$. Patients with immunosuppressed status, such as $\mathrm{HIV}$-infected, postchemotherapy cancer patients are particularly vulnerable to TB. According to CDC, $87.3 \%$ of reported TB cases in 2019 have known HIV status; among these $4.9 \%$ cases have coexisting HIV infection $(25,26)$.

As a cause of CNS infection, intracranial TB is usually acquired hematogenous from the extracranial sources, most frequently in the GI tract or pulmonary system. It is estimated that about $10 \%$ of $\mathrm{TB}$ cases present as CNS infections with $60-70 \%$ of those occurring in children and young adults under 20 years of age. Tuberculosis infection in the brain manifests in two major ways: TB meningitis and one or multiple caseating granulomas (tuberculomas). Meningitis accounts about $70-80 \%$ of CNS presentations and tuberculomas account for nearly all of the remaining 20-30\%. Rarely, CNS tuberculosis can present as an abscess $(18,26)$. CNS TB infection has a mortality of $25-30 \%$.

The typically symptoms of TB meningitis are like those 

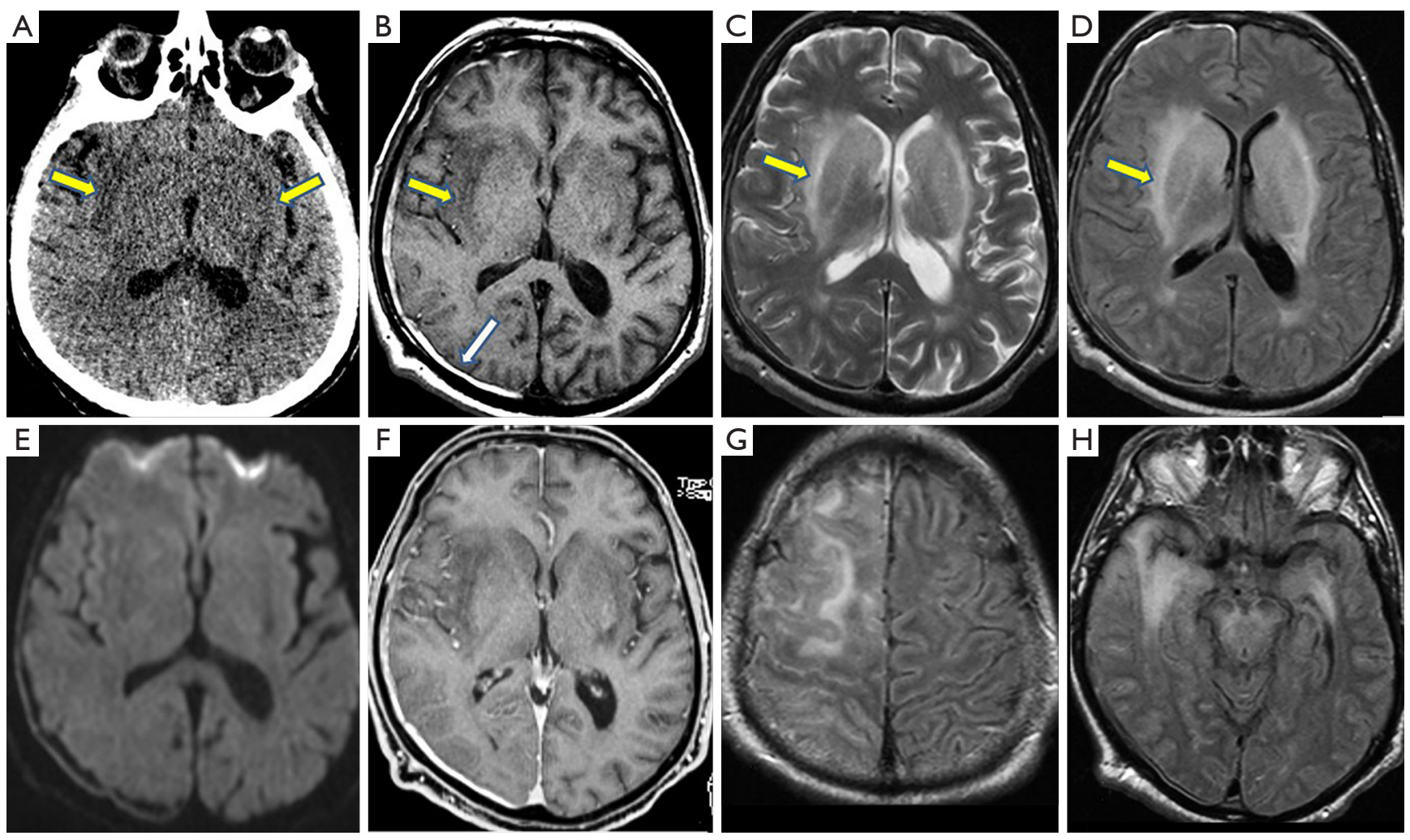

Figure 4 Eastern equine encephalitis (EEE). A 74-year-old male who had been working on an outdoor grill for a few days, was found to have fallen to the ground and passed out. He was brought to ER by EMS. Initial NECT of head was normal (not shown). He soon developed a fever of 102.4 F and left sided weakness. LP and further imaging followed. Axial NECT image (A) showed heterogenous hypodensities within bilateral basal ganglia and thalami, more at the external capsules (yellow arrow); On MR, axial T1 image (B) showed significant swelling of bilateral basal ganglia and thalami which appear heterogeneously hypointense, worse at bilateral external capsules. Incidentally, there is a small acute subdural hematoma (blue arrow) along the convexity of the right cerebral hemisphere secondary to the recent fall. Heterogeneously hyperintense signals were present in the same regions on axial T2 and FLAIR images (C,D). The hyperintensity of bilateral external and internal capsules were more conspicuous, corresponding the proposed "parenthesis sign". There was effacement of the lateral ventricles, worse on the right. No restricted diffusion or obvious enhancement was present on axial DWI (E) and post contrast axial T1 image (F). Axial FLAIR images (G,H) showed abnormal signals in the brainstem as well as asymmetrical involvement of the cortex. The patient's serology testing was positive for EEE. The patient's condition deteriorated quickly and eventually deceased after being comatose for 4 weeks without improvement. (All images of this case are from the Hospitals of the University of Massachusetts School of Medicine at Baystate Health System.) ER, emergency room; EMS, emergency medical services; NECT, nonenhanced CT; FLAIR, fluid-attenuation inversion recovery; DWI, diffusion weighted images.

observed in bacterial meningitis: nuchal rigidity, fever, headaches, vomiting, but in a subacute form and progressing gradually. Other findings include altered mental status, personality changes, cranial nerve palsies, ..., depending on the location or forms of infection (focal tuberculoma vs. meningeal involvement), with its severity ranging from headaches and mild meningeal symptoms to seizures and coma.

Diagnosis of CNS tuberculosis is challenging due to suboptimal sensitivity and specificity of diagnostic tests.
A definitive TB diagnosis in extra-neural site can be a strong indicator for CNS TB. LP should be avoided if there is concern for elevated intracranial pressure and risk of brainstem herniation. When CSF could be obtained, it typically shows lymphocytic pleocytosis, elevated protein, and decreased glucose. Microscopic proof of acid-fast bacilli (AFB) in CSF can give immediate result but usually require a large volume; Positive CSF culture for M. tuberculosis can be seen in less than $50 \%$ cases; the nucleic acid amplification 
test (NAAT) of CSF must be used in combination with AFB smear and culture. It has a rapid turnaround time with high sensitivity and specificity. But it has not been approved by the US Food and Drug Administration. A definitive diagnosis of tuberculoma is established by brain biopsy for histopathology and AFB stain and culture. Many times, a presumptive diagnosis is made for initiation of treatment in the setting of relevant clinical and epidemiologic factors and typical CSF findings $(27,28)$.

In early stages of TB meningitis, NECT is normal in approximately $30 \%$ of cases. Typical manifestations of TB meningitis include hydrocephalus, basilar exudates, periventricular infarcts, and multiple tuberculomas. Hydrocephalus can be seen in up to $75 \%$ cases. Basilar exudates commonly appear iso- or hyperdense on NECT which cause effacement of the related sulci and basal cisterns. On MR, these thick proteinaceous exudates demonstrate hypo or iso-intense T1 signals and hyperintense FLAIR signals. Contrast enhanced CT or MR may show uniform and intense enhancement along basilar meninges. Acute cerebral infarcts may occur in up to $50 \%$ patients with advanced disease and mostly involving basal ganglia $(18,27,28)$.

On CT, tuberculomas appear as as one or more round or lobulated hypo- or iso-dense lesions with edema out of proportion to mass effect but little enhancement in early stages and encapsulated hyperdense lesions with ring enhancement on late stages. These vary widely in size and most are less than $2.5 \mathrm{~cm}$ in diameter. They may demonstrate central calcification in chronic lesions. On MR, tuberculomas appear as discrete, single or multiple hypointense T1 and hyperintense T2 lesions with ring or homogeneous disc-shaped enhancement. Larger tuberculomas with necrotic centers may show restricted diffusion within the fluid cavities on DWI. They usually exert considerable surrounding edema and mass effect (18). Sometimes, innumerable small tuberculomas of a few millimeters in sizes can be observed in the brain of patients with a military TB (Figure 5).

Differential diagnosis of TB meningitis includes other pyogenic meningitis, especially those with predilection to basal cisterns such as Mycobacterium avium infection (MAI), syphilis or other granulomatous infections. Meningeal carcinomatosis is another common differential diagnosis if patient has a known neoplasm elsewhere. The differential diagnoses of tuberculoma include neurocysticercosis, a pyogenic abscess and a primary or metastatic neoplasm differentials.
The classic RIPE (rifampin, isoniazid, pyrazinamide, ethambutol) therapy is the first-line treatment for tuberculosis. Generally, 12 months of antibiotic therapy is considered adequate to eliminate even intracranial tuberculomas. The CDC also recommends dexamethasone for 6-8 weeks for management of tuberculous meningitis. In cases of hydrocephalus that were noncommunicating or resistant to diuretic therapy, the placement of a ventriculoperitoneal shunt is effective in relieving intracranial pressure and improving outcomes even in severe cases.

\section{Extra-axial empyema}

Intracranial empyemas have two types: epidural empyema and subdural empyema. Epidural empyema is also called epidural abscess, a collection of pus between the dura matter and the calvarium. Subdural empyema is a collection of pus between the arachnoid matter and the dura matter. Epidural empyemas have biconvex shapes that are confined by sutures but can cross midline by dissecting across dural sinuses. Subdural empyemas have crescent shapes that are not confined by sutures but limited by falx cerebri, tentorium cerebelli, and foreman magnum. It otherwise can spread freely to adopt the convexity of the cerebral hemispheres or line the interhemispheric fissure. Both types of empyemas can occur at any age though more common in adults, with no gender predilection and are responsible for $20-33 \%$ of all intracranial infection (5).

In older children and adults, the empyemas are predominantly acquired through direct spread from an adjacent infectious focus, such as paranasal sinusitis, otitis media, mastoiditis, or skull OM, while in infants, the leading cause of empyemas are pyogenic meningitis. Alternatively, distal infections can seed into these potential extra axial spaces through hematogenous transmission or can track up the CSF flow from spinal infection. Subdural empyema was also reported as a complication following invasive procedures such as evacuation of chronic subdural hematomas, and as such can be confused with a recurrent subdural hematoma without advanced neuroimaging (29-31). Causative organisms are dependent on the initial source of infection; in infants, the group B streptococci, E. coli and listeria monocytogenes are the most common causes of pyogenic meningitis; while in older population the isolated culprits include aerobic and anaerobic streptococci, stapbylococci, aerobic gram-negative bacilli, anaerobes, and fungi $(5,18)$.

Diagnosing extra-axial empyema is difficult based on clinical findings alone due to nonspecific presenting 

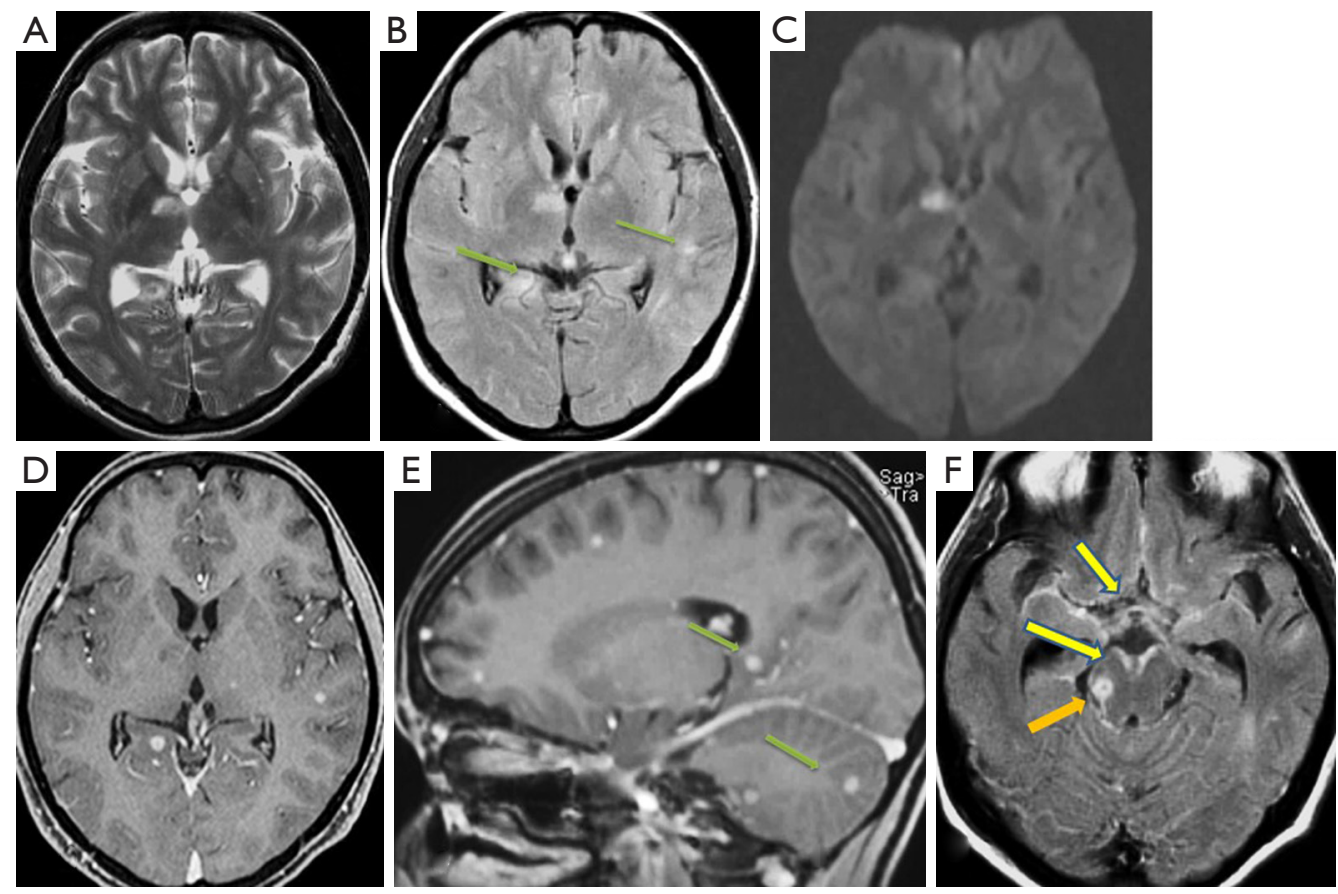

Figure 5 CNS tuberculosis. A 26-year-old previously healthy female recently came from Nepal presented with one month of headaches and weight loss. She also developed low grade fever, chills, neck pain, nausea and vomiting. On MR, axial T1 image (not shown) was unremarkable. Axial T2 and FLAIR images (A,B) showed multiple small solid and ring-like hyperintense foci throughout the brain. One of the lesions in the right basal ganglia demonstrated restricted diffusion on axial DWI (C) but no enhancement on post contrast axial T1 image (D). This could represent a focus of acute infection. Other hyperintense lesions (green arrows) showed no restricted diffusion but had solid or rim enhancement. Postcontrast sag T1 image (E) showed numerous enhancing nodules throughout the supratentorial and infratentorial brain which could represent tuberculomas. The patient's CSF RNA testing was positive for TB. Axial postcontrast FLAIR image (F) of a companion case showed TB meningitis concentrated at the basilar cisterns (yellow arrows), with a tuberculoma in the right cerebral peduncle (orange arrow). (All images of this case are from the Hospitals of the University of Massachusetts School of Medicine at Baystate Health System.) CNS, central nervous system; FLAIR, fluid-attenuation inversion recovery; DWI, diffusion weighted images; CSF, cerebral spinal fluid; TB, tuberculosis.

symptoms and laboratory tests. Fever, headache, lethargy, vomiting, and seizures, in combination with sinusitis, otitis media, mastoiditis, recent neurosurgery or head trauma should raise clinical suspicion. LP is not indicated as it provides little useful information and may precipitate brain herniation. Early diagnosis of extra axial empyema is crucial as patient may progress rapidly to coma or death.

Neuroimaging findings often provide more definite diagnosis. NECT is the gateway imaging modality that may show abnormal epidural or subdural fluid collections though subtle cases can be missed. The purulent materials may be hypodense to isodense to brain on NECT. Subtle effacement of the underlying cortical sulci or mild compression of the ventricle hints at mass effect in less obvious cases. On CECT, there is diffuse enhancement along the periphery of the fluid collections. MRI is the imaging modality of choice for evaluating empyemas. On none-enhanced MRI, empyema shows an equal inconspicuousness on T1WIhypointense to isointense to the brain parenchyma, and mildly hyperintense to CSF. On T2WI and FLAIR, empyema gives off heterogeneous high intensity signals. Markedly restricted diffusion of the stagnant pus in extra axial spaces are the characteristic and crucial findings of empyemas, differentiating them from other nonrestricted extra-axial collections like subdural effusion, hygroma, or hematoma. Many times, the adjacent brain parenchyma shows similar hypointense $\mathrm{T} 1$ and hyperintense T2/ FLAIR signals with restricted diffusion indicating associated encephalitis or ischemia. After intravenous contrast administration, the granulomatous or fibrous 

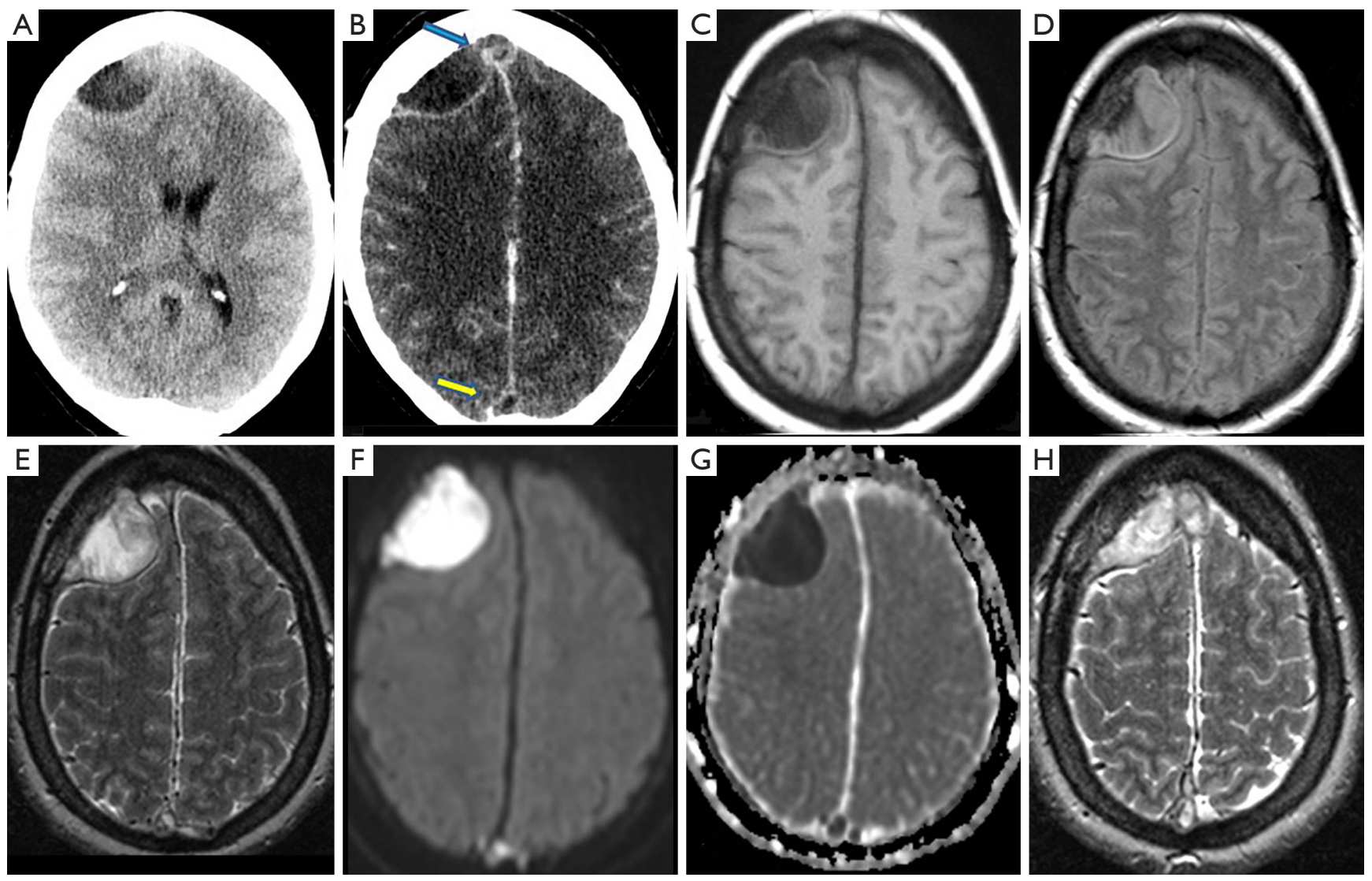

Figure 6 Epidural empyema. A 32-year-old pregnant women who was on Amoxil treating sinusitis for several weeks, presented to ER with intractable headaches, fever, chills, nausea, vomiting and stroke like symptoms. Axial NECT (A) showed an epidural fluid collection with mixed hypo- and hyperdensities, associated with effacement of the right frontal horn and mild right to left midline shift. This epidural collection showed diffuse rim enhancement on the axial CECT image (B), which extended into the superior sagittal sinus (SSS) causing thrombosis (blue arrow). Note the thrombosis within the posterior portion of the SSS (yellow arrow), consistent with a "delta sign". On MR, the epidural collection appeared hypointense on axial T1 image (C) and heterogeneously hyperintense on axial T2 and FLAIR images (D,E). It demonstrated significant restricted diffusion on axial DWI and ADC images (F,G), consistent with an epidural empyema. Higher cut axial T2 image showed extension of the epidural collection into the SSS. Staphylococcal species and Candida Albicans were identified from drainage materials. (All images of this case are from the Hospitals of the University of Massachusetts School of Medicine at Baystate Health System.) NECT, nonenhanced CT; CECT, contrast-enhanced computed tomography; DWI, diffusion weighted images; ADC, apparent diffusion coefficient.

capsules surrounding the empyemas demonstrate diffuse meningeal enhancement in both CT and MR (32-34). Differentiation of subdural and epidural empyemas is based on its locations and morphological appearance, as to whether the collection is bounded by calvarial sutures or falx (Figures 6,7).

The epidural or subdural hematomas are the main differential diagnoses for epidural or subdural empyema respectively. On NECT, acute hemorrhages appear hyperdense. This hyperdensity gradually decreases over time. MRI signal characteristics of hemorrhages change over time as well. From acute to subacute stages, the hematoma changes from iso/hypo- intense to hyperintense on T1WI and iso/hyper- intense to hyperintense on T2 WI. From the subacute to chronic stages, it appears hyperintense on both T1WI and T2WI. Another differential diagnosis is subdural effusion which is usually a complication of meningitis. Its appearance and location are similar to the subdural empyema, but the fluid remains hypodense on CT and hypointense on T1WI of MR without enhancement. It 

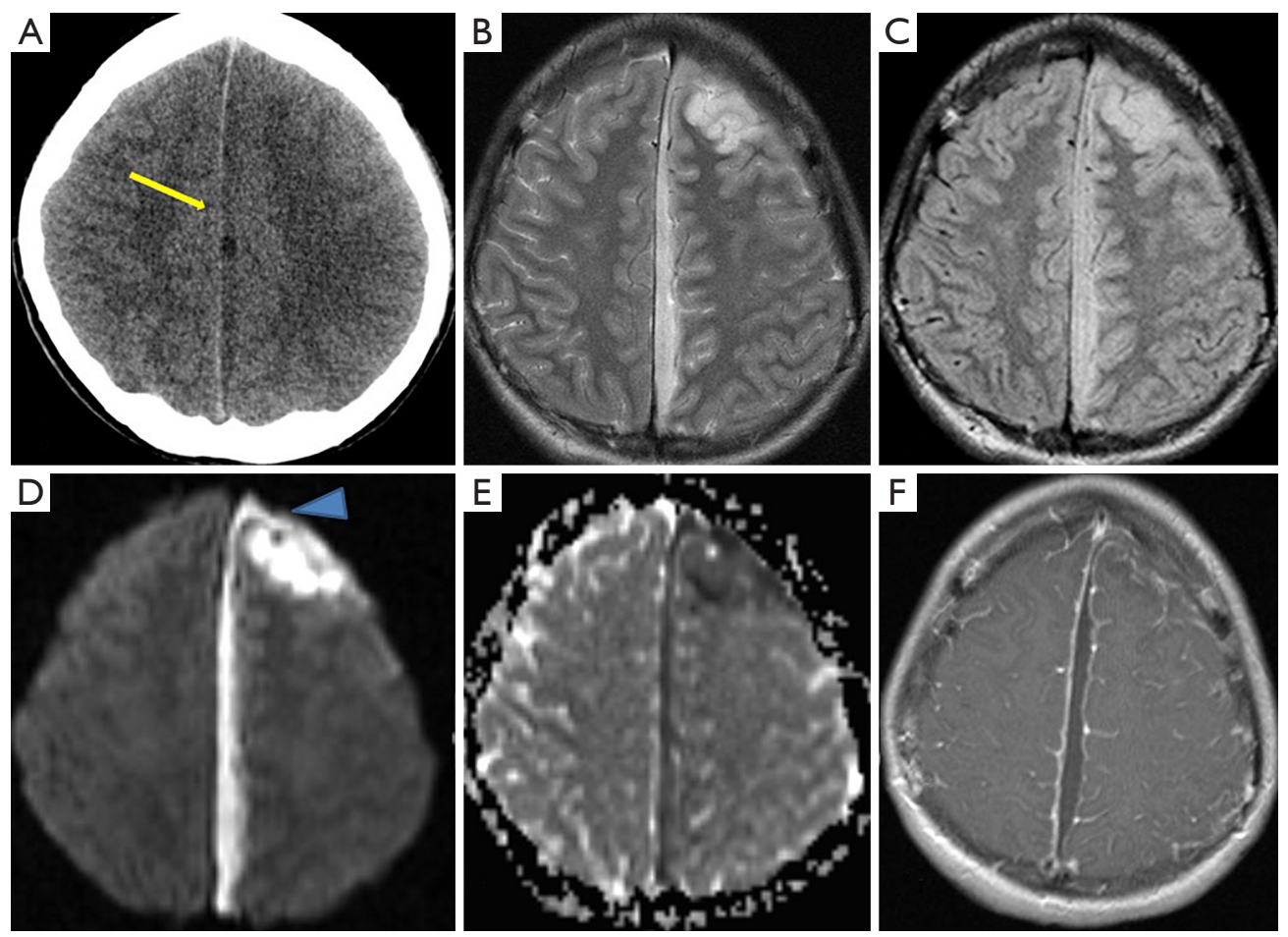

Figure 7 Subdural empyema. A 13-year-old boy with one-week history of cold and sinusitis, presented to ER with fever, headaches, vomiting and malaise for 2 days. He developed right lower leg numbness on the day of admission. Axial NECT (A) showed an iso- to hypodense subdural fluid collection along the left sided falx (arrow). On MR, this fluid collection appeared hyperintense on axial T2 and FLAIR images (B,C) and demonstrated restricted diffusion on axial DWI and ADC image (D,E). It extended to the left anterior frontal subdural space (arrowhead). On postcontrast axial T1 image (F), there was diffuse enhancement along the periphery of the fluid collection, consistent with a subdural empyema. Diffuse leptomeningeal enhancement is also noted along the sulci of bilateral frontoparietal lobes (worse on the left), consistent with meningitis. The left anterior frontal lobe contained a focal area of hyperintense T2/FLAIR signals with restricted diffusion and heterogeneous enhancement which could be due to either cerebritis or vasogenic edema. Culture of drained empyema grew Streptococci viridans and Fusobacterium nucleatum. (All images of this case are from the Hospitals of the University of Massachusetts School of Medicine at Baystate Health System.) ER, emergency room; NECT, nonenhanced CT; DWI, diffusion weighted images; ADC, apparent diffusion coefficient; FLAIR, fluid-attenuation inversion recovery.

shows no restricted diffusion on DWI.

The major complications of empyema include cerebritis, abscess formation, infarct, and cortical vein or dural sinus thrombosis, which have been related to significantly worse prognoses. Mortality can be as high as $10-20 \%$ despite early diagnosis and appropriate treatment with surgical evacuation and aggressive antibiotic therapy $(5,32,33)$. Wide craniotomy is often preferred over Burr hole drainage, which has better clearance of empyema and improved clinical outcome.

\section{Brain abscess}

Brain abscess is a collection of pus surrounded by a wellvascularized capsule, enclosed in the brain tissues, usually as a result of a bacterial or fungal infection. Its development has 4 stages: at the first, the related brain tissues develop an acute infection, i.e., early cerebritis (1-4 days); second, the cerebritis progresses to "later cerebritis" (4-10 days), with the brain tissues become necrotic, starting to form a cavity containing purulent materials, i.e., a pyogenic abscess. The third stage (11-14 days) and the fourth stage ( $>14$ days) delineate the formation of a vascularized capsule surrounding the abscess (35). Brain abscess is a serious and potentially life-threatening condition. Without treatment, it is invariably fatal.

Brain abscess is an uncommon disease and its incidence varies among different countries. It accounts for $8 \%$ of intracranial masses in developing countries and $1-2 \%$ in 
western countries. In the United States, approximately 1,500-2,500 cases are diagnosed annually. Over the past three decades, mortality from brain abscess has decreased from $30-40 \%$ to $5-20 \%$, with the higher rates reported in developing nations. The highest incidence group is adult men below 30 years of age while children between ages 4-7 are the second highest group who often are associated with cyanotic heart diseases. About $25 \%$ of patients are less than 15 years of age. The affected male to female ratio is $2: 1$ to $3: 1$ for undetermined reasons $(35,36)$.

Like many other CNS infections, brain abscess can be acquired via two routes: hematogenous dissemination through septicemia from a distant extracranial source such as pulmonary infectious processes and urinary tract infection; or direct spread from nearby infectious focus such as paranasal sinusitis, mastoiditis, or otitis media. Other possibilities include complications of local head trauma or recent neurosurgical interventions. Brain abscesses associated with septicemia commonly cause multiple abscesses, mostly in the distribution of the middle cerebral artery (MCA) and usually at the gray-white matter junction. Direct spread of primary infection intracranially usually causes a single abscess in the contiguous brain tissues.

The most common etiology is bacterial, while fungal or parasitic etiologies are less common. Up to $30 \%$ of brain abscesses are reported with unknown origin. In immunocompetent patients, the most common causative microorganisms are Streptococcus species, Staphylococcus aureus and pneumococci. Klebsiella commonly affects diabetics while Aspergillus and Nocardia are common in transplant patients. Bacterial abscesses are less common in immunocompromised patients (37). Toxoplasmosis and Mycobacterium tuberculosis are most common in HIV/AIDS patients (see later sections).

The patients' clinical symptoms depend on the location of the abscess, degree of mass effect, and associated complications. Unfortunately, the most frequent presenting symptoms are nonspecific, including headache, fever, focal neurologic deficit, nausea, vomiting, and seizure.

Diagnosis is based on combination of clinical findings and neuroimaging results. Serology tests are nonspecific. LP is usually not helpful for diagnosis and often contraindicated if patients show papilledema or other focal symptoms or signs because of possible increased intracranial pressure.

Neuroimaging findings of cerebral abscesses vary depending on the stages of the abscesses and time of imaging. Early cerebritis appears as an irregular area of hypodensity that does not enhance following contrast injection. As cerebritis evolves, the hypodense lesion enlarges with thick and diffuse ring enhancement following contrast injection. The ring enhancement represents breakdown of the blood-brain barrier and the development of an inflammatory capsule. In the capsular stage, NECT shows a hypodense cavitating lesion with some dependent debris as well as surrounding vasogenic edema and mass effect. Post contrast CT shows a thick ring or nodular rim enhancement. The rim enhancement may not be uniform in thickness and is often less prominent at the medial or ventricular surface of the abscess (36-38). On MR, the typical findings of an abscess, i.e., in the capsular stage, are a cystic lesion with central hypointense T1 and hyperintense T2/FLAIR signals. The capsule may be visible as a hypointense or intermediate rim on T2/FLAIR images. On the susceptibility images, there may be a hypointense rim suggesting hemorrhages. The surrounding vasogenic edema shows irregular hypointense $\mathrm{T} 1$ and hyperintense T2/FLAIR signals. The marked hyperintensity of pus in the necrotic center on DWI with corresponding hypointense signals on ADC map is the characteristic finding of a pyogenic brain abscess $(38,39)$. On postcontrast MRI, a smooth thin continuous ring is most suggestive of an abscess (Figure 8).

Major differential considerations include cystic neoplasms and tumefactive multiple sclerosis (MS). Most cystic neoplasms of the brain will not demonstrate restricted diffusion aside from dermoid tumors and CNS lymphoma. Additionally, clinical history will be highly important with symptoms of fever, headache, and leukocytosis raising much higher concern for abscess versus tumor. Tumefactive MS is a rare form of MS in which the MS plaque shows a "tumorlike" appearance with its size greater than $2 \mathrm{~cm}$, associated with surrounding edema and mass effect. It usually has a hypointense $\mathrm{T} 2 \mathrm{rim}$ and incomplete ring enhancement. Multiple smaller but classical MS plaques are often seen in the rest of the white matters. Patient's clinical history also provide additional clue for differential diagnosis.

Complications of brain abscesses are various, including local mass effect with intracranial herniation, hydrocephalus, meningitis, extra-axial empyema and venous/dural sinus thrombosis. Successful treatment depends on a combination of intravenous antibiotics and neurological intervention for abscess drainage. A target-specific antibiotic is applied when the culprit is known. Otherwise, a broad-spectrum antibiotic is employed. Radiology imaging is paramount for the early detection of a brain abscess and evaluation for its complications as well as monitoring its treatment. 

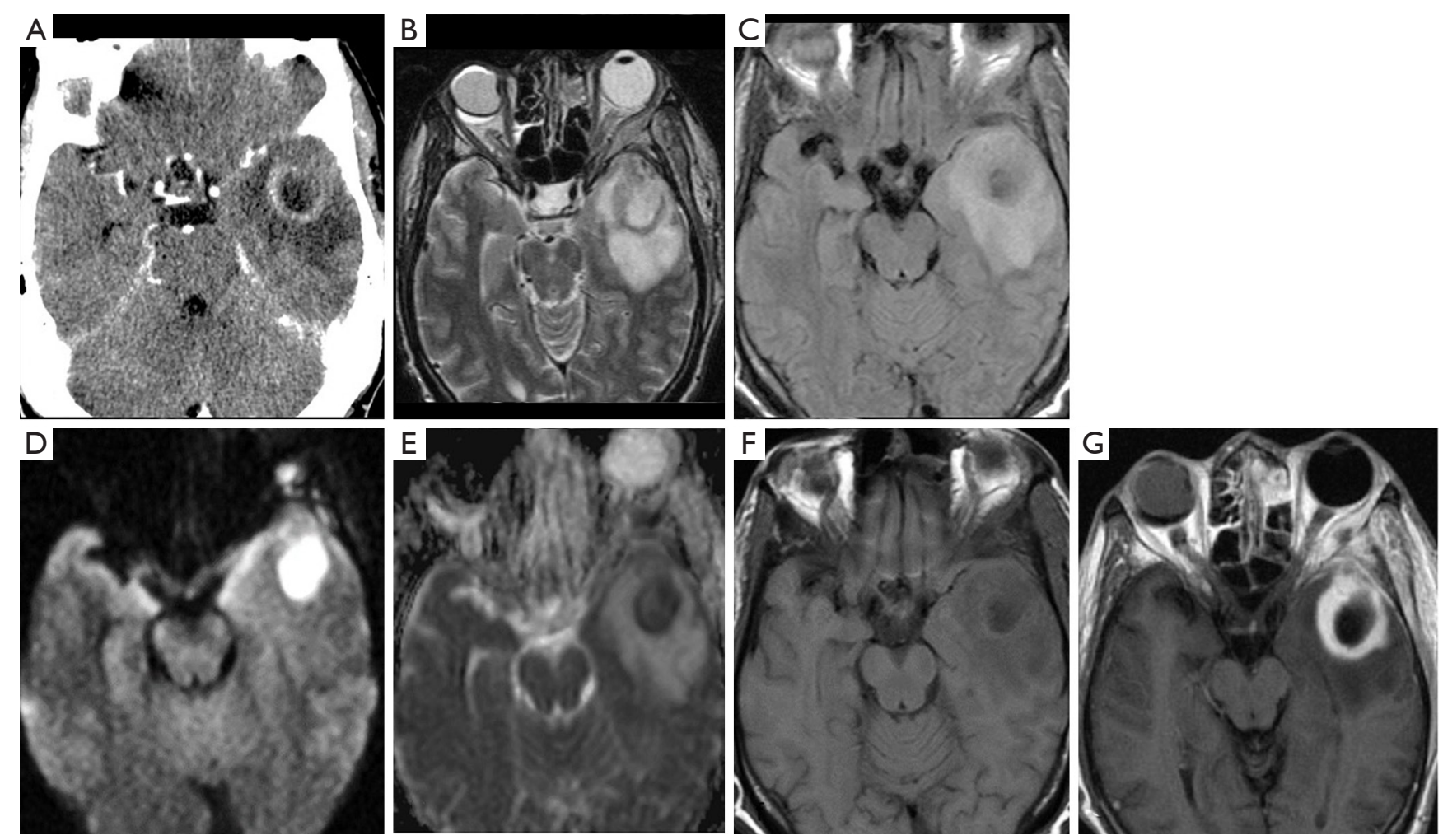

Figure 8 Pyogenic brain abscess. A 55 -year-old male presented with fever of $102.5 \mathrm{~F}$, chills, left frontal headaches and left eye swelling. NECT of head showed severe sinusitis of the left ethmoid and maxillary sinuses (not shown). Axial CECT image (A) showed a cystic lesion with incomplete rim enhancement in the left anterior temporal lobe, associated with surrounding edema. On MR, this lesion had a hypointense rim and hyperintense center on axial T2 image (B) as well as an isointense rim and hypointense center on axial FLAIR image (C). The surrounding hyperintense T2/FLAIR signals were consistent with vasogenic edema. The central contents of this cystic lesion demonstrated restricted diffusion on axial DWI and ADC images (D,E), suggesting purulent materials. Pre- and postcontrast axial T1 images (F,G) showed thick rim-enhancement of the lesion. The findings were consistent with a pyogenic abscess. Streptococcus Viridans was identified in the culture. (All images of this case are from the Hospitals of the University of Massachusetts School of Medicine at Baystate Health System.) CECT, contrast-enhanced computed tomography; FLAIR, fluid-attenuation inversion recovery; DWI, diffusion weighted images; ADC, apparent diffusion coefficient.

\section{Ventriculitis/ependymitis}

Ventriculitis refers to infection and inflammation of the ventricles including and its contents including ventriculitis, ependymitis, ventricular empyema, and pyocephalus. Specifically, ependymitis refers to the diseased ependymal lining. Ventriculitis is an uncommon complication of severe intracranial infections, such as meningitis, encephalitis or brain abscesses. It is the result of the spread of pyogenic infections into the ventricles or along the ependyma. The routes of transmission include extension of preexisting meningitis/encephalitis to the ventricles via the subependymal or choroid plexus, rupture of brain abscess, or contiguous extension from trauma or neurosurgical interventions such as an external ventricular drainage. Ventriculitis is estimated to occur in approximately $30 \%$ of patients with meningitis. It is more often seen in neonatal meningitis which occurs in $20 \%$ of cases but comprises $80 \%$ to $90 \%$ of all patients with ventriculitis. Staphylococcus and Enterobacter are the two most common causative microorganisms (40).

Besides the clinical manifestations of the primary infections, such as meningoencephalitis or pyogenic abscess, there are no reliable clinical signs of ventriculitis. Nonresponsive to antibiotics may raise a high index of suspicion. Blood tests are usually nonspecific. Gram stain or culture of CSF can be done and may be positive if 

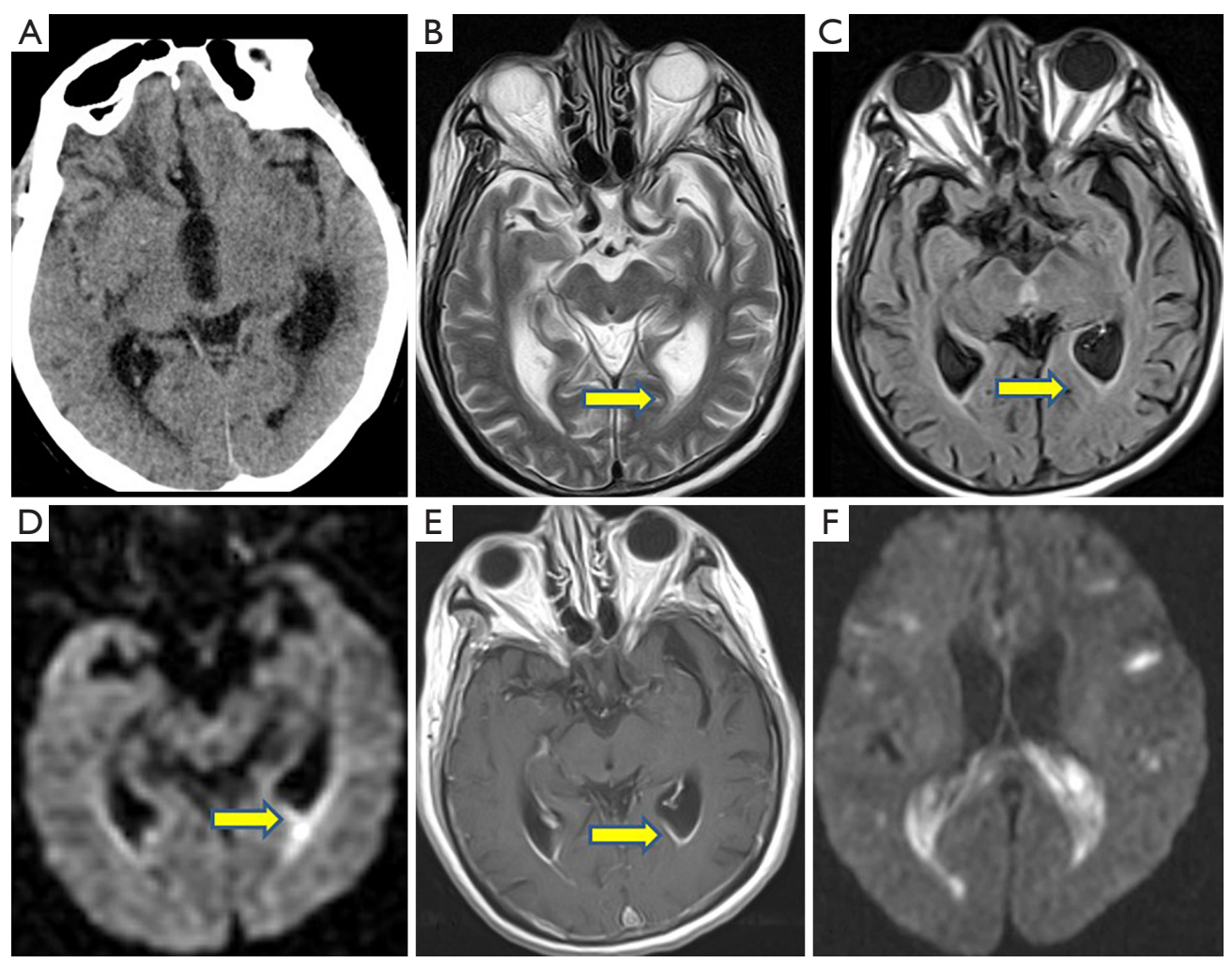

Figure 9 Ventriculitis/ependymitis. A 77-year-old female presented to ER with lethargy, urinary tract infection and seizure-like activity. Her blood sugar was in 700's. Further evaluation revealed liver abscesses. Axial NECT (A) showed hydrocephalus and mild periventricular hypodensities. An old infarct was seen in the right frontal lobe. On MR, axial T2 and FLAIR images (B,C) showed mild hyperintense signals in the periventricular white matters along the ependymal lining of the ventricles, which demonstrated restricted diffusion on axial DWI image (D) and enhancement on post contrast axial T1 image (D). The findings were consistent with ventriculitis. A few days later, hyperintense signals were seen in the dependent areas of the lateral ventricles as well as the sulci of bilateral cerebral hemispheres on axial DWI image (F) suggesting accumulation of pus. Surgical drainage grew Klebsiella pneumoniae. (All images of this case are from the Hospitals of the University of Massachusetts School of Medicine at Baystate Health System.) ER, emergency room; NECT, nonenhanced CT; FLAIR, fluid-attenuation inversion recovery; DWI, diffusion weighted images.

drainage is required for obstructive hydrocephalus. Other CSF findings are nonspecific for infection: leukocytosis, decreased glucose and elevated protein levels. Increased intracranial pressure is usually present.

The diagnosis of ventriculitis is challenging as the clinical pictures are nonspecific but devastating. NECT may be normal or show periventricular hypodensities which could be due to periventricular inflammatory changes or transependymal fluids. Suppurative debris layering in the dependent areas of ventricles appears hyperdense and is the most characteristic finding in one series. Ependymal enhancement may possibly be seen on contrast enhanced CT. Characteristic MRI imaging includes restricted diffusivity on DWI evident within the dependent debris or along the ependymal surface. The layering debris in the occipital horns is T1 hyperintense relative to CSF and T2 hypointense. A periventricular halo of FLAIR/T2 as well as focal or diffuse enhancement along the ventricles is highly suggestive of ependymitis. Restricted diffusion on DWI along the ependymal surface or in the dependent areas of the ventricles are strong evidence of ventriculitis/ ependymitis and accumulated pus. Sometimes, a poorly marginated swollen and enhancing choroid plexus is noted, consistent with choroid plexitis. This could be another characteristic MRI finding of ventriculitis $(40,41)$ (Figure 9).

Differential diagnoses of ventriculitis on imaging include subarachnoid or ependymal tumor seeding and intraventricular hemorrhages. Although subarachnoid 
or ependymal tumor seeding may show ependymal enhancement but hyperintense diffusion signals along the ventricles generally separate them from ventriculitis/ ependymitis. The clots of intraventricular hemorrhages also do not show restricted diffusion on DWI.

Ventriculitis/ependymitis is associated with high morbidity and mortality. Early detection and treatment offer the best hope for survival. The mainstay of treatment is intravenous antimicrobial therapy which can reach effective concentrations in CSF. Specific antibiotics are chosen based on CSF culture and penetration into CSF when meningeal inflammation is present. For catheter-related ventriculitis, removal of all components of the infected shunt or EVD, in combination with antimicrobial therapy is recommended.

\section{Septic-embolic encephalitis (SEE)}

SEE is caused by infected thromboemboli occluding the blood vessels of the brain. The affected brain manifests with two attributes of the disease: an ischemic insult due to vascular occlusion and an infectious insult from a deepseated infection nidus. The ischemia may lead to brain infarcts; The infection may develop into brain abscesses. Mycotic aneurysms can also develop from the locations of septic emboli, which account for less than $10 \%$ neurological complications of infective endocarditis (IE). Intracranial hemorrhages could be complications of ischemic stroke, brain abscesses or rupture of a mycotic aneurysm $(42,43)$.

SEE is usually a part of a systemic infection with septic emboli in multiple organs of the body, and the brain being the most common location. The most common origin of septic emboli is IE. Staphylococcus aureus is the leading causative microorganism, followed by Streptococcus viridian, enterococci and coagulase-negative staphylococci. IE patients with vegetation size $>3 \mathrm{~cm}$, Staphylococcus aureus infection, mitral valve involvement, and anticoagulation therapy are at higher risks of septic emboli. Other commonly reported sources of septic emboli include sepsis from intravenous drug use, infection of instrumental devices such as indwelling catheter or pacer wires, pulmonary abscess, etc. (44-46).

Symptoms of SEE vary from asymptomatic to severe with a high mortality. Ischemic stroke is the most common presenting symptom of SEE; $15 \%$ of patients present with transient ischemia. Neurological deficits depend on the location and extend of the disease. Other symptoms include altered mental status, psychosis, headaches, etc.

Serology tests show signs of systemic infection such as leukocytosis, elevated ESR, etc. The inciting pathogen may be identified by gram stain. Blood culture is almost always positive. LP is not usually performed as it provides little useful information. Onset of neurologic deficits in patients with a systemic infection should raise a high index of suspicion for SEE. Positive blood culture and neuroimaging findings point to a more definitive diagnosis.

Neuroimaging findings of SEE are commonly composed of ischemic infarct and brain abscesses. The ischemic infarct mostly occurs in the distribution of MCA and rarely in posterior circulation. Among IE patients, $20-55 \%$ present symptomatically with ischemic or hemorrhagic stroke; $60-80 \%$ are asymptomatic but show positive findings on brain MRI. Abscesses caused by septic emboli are usually multiple, small and of various ages, different than the brain abscesses described in the earlier section of this article. They could be seen in multiple vascular distributions, but mostly concentrated along the peripheral watershed territory or gray-white interface. If manifesting as cerebral microbleeds, the lesions are preferentially found in cortical areas (42-44). NECT scans show multiple low-attenuation foci, with or without microhemorrhage. Punctate or ring enhancement is noted after contrast administration. MR scans show multiple abscesses of various sizes in the brain-multiple cavitating lesions with rim-enhancement. The central lumen is associated with restricted diffusion. Hemorrhagic lesions can be correlated with "blooming" artifacts on susceptibility sequences. Similar to CT, contrast enhanced $\mathrm{T} 1$ images often demonstrate punctate or ring enhancement, similar to micro abscesses (Figure 10).

The main differential diagnoses include metastasis and MS. The images of metastasis show multiple round well-circumscribed ring-enhancing lesions which are often similar in sizes and typically locate in or near graywhite matter junctions. They are usually associated with significant vasogenic edema but lack of central restricted diffusion. MS usually has a long relapsing and remitting course, commonly present with typical imaging findings such as periventricular Dawson's fingers, corpus callosum lesions, etc. The enhancement of MS plaques varies from solid to incomplete ring enhancement which only occurs in active demyelinating phase.

Management of SEE requires a multidisciplinary approach. Early organism-specific antibiotics are always recommended unless cryptogenic in which case a broad-spectrum antibiotic is recruited. Anticoagulation and thrombolysis are not therapeutic and should be avoided due to complications. Endovascular intervention can be helpful in case of acute septic emboli or mycotic aneurysm. Early cardiac surgery for 

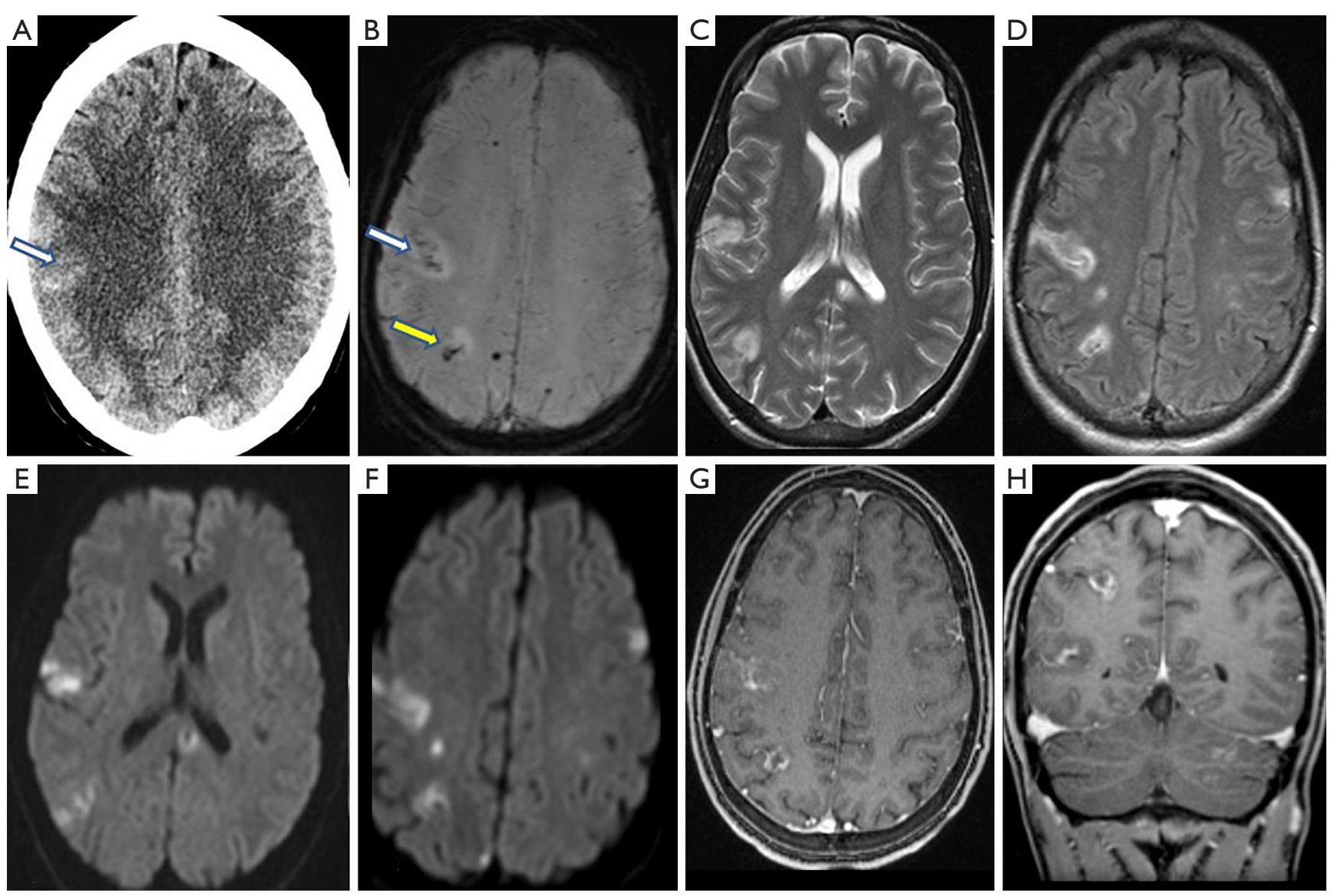

Figure 10 Septic embolic encephalitis. A 26-year-old IV drug abuser with history of endocarditis and recent surgery for mitral valve replacement, presented with 2 days of fever, fatigue and imbalance. Initial axial NECT (A) showed scattered areas of acute subarachnoid hemorrhages (blue arrow). CTA of head was unyielding (not shown). On subsequent MR, axial GRE image (B) showed not only subarachnoid hemorrhages but also foci of intraparenchymal hemorrhages (yellow arrow). Axial T2 and FLAIR images (C,D) showed multiple irregular areas of hyperintense signals throughout the brain, which demonstrated restricted diffusion on axial DWI images (E,F), consistent with acute embolic infarcts. On post contrast axial T1 images, these lesions are associated with underlying heterogeneous enhancement. The patient's blood culture grew MRSA. The findings are consistent with septic emboli. (All images of this case are from the Hospitals of the University of Massachusetts School of Medicine at Baystate Health System.) IV, intraveneous; NECT, nonenhanced CT; CTA, computed tomography angiography; GRE, gradient echo sequence; FLAIR, fluid-attenuation inversion recovery; DWI, diffusion weighted images; MRSA, methicillin-resistant Staphylococcus aureus.

endocarditis is proven safe in the setting of recent cerebral ischemic events (42). Current guidelines recommend surgery to prevent recurrent embolism in high-risk patients or prophylactic surgical intervention for the patients with vegetation $>10 \mathrm{~mm}$ with severe valvular dysfunction.

\section{Human immunodeficiency virus (HIV)-associated opportunistic infections of the CNS}

HIV damages the immune system of the human body, making it vulnerable to infections and neoplasms. Most of these opportunistic infections were rare in the pre-HIV epidemic era and manifested due to the immunocompromised status of HIV patients. Acquired immunodeficiency syndrome (AIDS) is a more advanced stage of HIV infection. Well-known CNS infections in this population include toxoplasmosis, cryptococcosis, progressive multifocal leukoencephalopathy (PML), cytomegalovirus encephalitis, neurosyphilis, and tuberculosis. In the late era of highly active or combined 
antiretroviral therapy (HAART or cART), opportunistic CNS infections in HIV-positive patients have declined drastically. In a retrospective study, cerebral lesions were detected in $21.8 \%$ of patients in the pre-HAART era versus $7.6 \%$ in the early HAART era. (47). Lack of access to HAART or cART, with persistent low T-lymphcyte (CD4) cell count, has been associated with higher risk of CNS infections. A small percentage of these patients may experience immune reconstitution inflammatory syndrome (IRIS)-paradoxical worsening of clinical condition following initiation of HAART, in setting of active infection. HAART should be held for at least two weeks to reduce the risk of IRIS.

\section{A. Toxoplasma encephalitis (TE)}

TE or cerebral toxoplasmosis caused by toxoplasma gondii-an obligate intracellular protozoan-is the most common CNS infection in AIDS patients and the leading cause of death within this population. Its incidence is $15-50 \%$, increased to about $30 \%$ when their CD $4<100$. Studies have emphasized that the importance of continued HAART and prophylactic treatments could reduce the risk of TE by $50 \%$ when patients are on antiretroviral therapy and $53 \%$ when patients are mainly treated with prophylaxis $(47,48)$.

Clinical symptoms TE is vague and indolent. The most common presenting symptom is headache, often accompanied by altered mental status and fever. Movement disorder and ataxia should raise suspicion for toxoplasmosis, correlating to radiologic or postmortem findings of brain abscesses most commonly involving the basal ganglia and the corticomedullary junction. Other nonspecific symptoms include but are not limited to dementia, ataxia, lethargy, and seizures. A presumptive diagnosis can be made if the patient has a CD4 count $<100$ cells/mL, in combination of compatible clinical symptoms, positive serological antitoxopalsma IgG antibodies and typical neuroimaging findings. Definite diagnosis is made with detection of DNA of toxoplasma by PCR in CSF (50-98\% sensitivity; 96-100\% specificity), with brain biopsy required in certain cases (49).

Neuroimaging findings are not pathognomonic, but some features are more commonly present. On NECT, TE typically manifests as multiple hypodense lesions associated with significant vasogenic edema, often with a predilection for the basal ganglia and thalami. Corticomedullary junction is another common location. Calcifications are not present except in congenital TE. Thin smooth rim enhancement or sometimes eccentric nodular enhancement can be seen after intravenous contrast administration, which becomes more conspicuous with higher white count, and usually absent with CD $4<50$ cells/cc (50). On MRI, TE demonstrates multiple focal lesions with hypointense $\mathrm{T} 1$ and hyperintense or mixed T2 signals, in the similar locations within the brain. These lesions may have hyperintense T1 signals along the rim, presumably representing coagulative necrosis or hemorrhage. Typical ring enhancement is seen in $90 \%$ cases. Nodular enhancement is sometimes noted. At times, a small eccentric nodule abutting an enhancing ring (i.e., inflamed vascular zone surrounding the necrotic abscess) was called a "target sign" and was thought to be highly suggestive of toxoplasmosis. Marked perilesional edema appear hypointense on T1 and hyperintense on T2/FLAIR images. On DWI, the core tissues of ring-enhancing toxoplasma abscesses usually show no restricted diffusion, unlike pyogenic abscesses. The peripheral hyperintensity on DWI may represent the presence of hemorrhage within their walls or due to $\mathrm{T} 2$ shine-through phenomenon of associated edema (51,52) (Figure 11).

The differential diagnosis of multiple ring enhancing lesions in the brain is extensive. The most common ones are metastatic disease and septic emboli among the nonimmunocompromised patients and lymphoma among the immunodeficient patient. Metastatic disease often has a known history of tumor and presents as multiple round well-circumscribed ring-enhancing lesions which are often similar in sizes and typically locate in or near gray-white matter junctions. They are usually associated with significant vasogenic edema but lack of central restricted diffusion. Ring enhancing lesions of SEE tend to locate at corticomedullary junction, more commonly in MCA distribution without predilection to basal ganglia. Emboli also cause acute infarcts in the associated vessel distribution/territory which are not seen in toxoplasmosis or metastatic disease. The infection can cause multiple abscesses of various sizes in the brain with central lumen associated with restricted diffusion. Lymphoma may sometimes manifest as ring enhancing lesions. But their sizes are usually larger than those in TE, and more infiltrative in appearance and more heterogeneous in enhancement. They typically show restricted diffusion. The corpus callosum and periventricular areas are more commonly involved (49,51).

The treatment of TE comprise antimicrobial therapy directed against $T$. gondii and antiretroviral therapy for immune recovery. A combination of pyrimethamine 

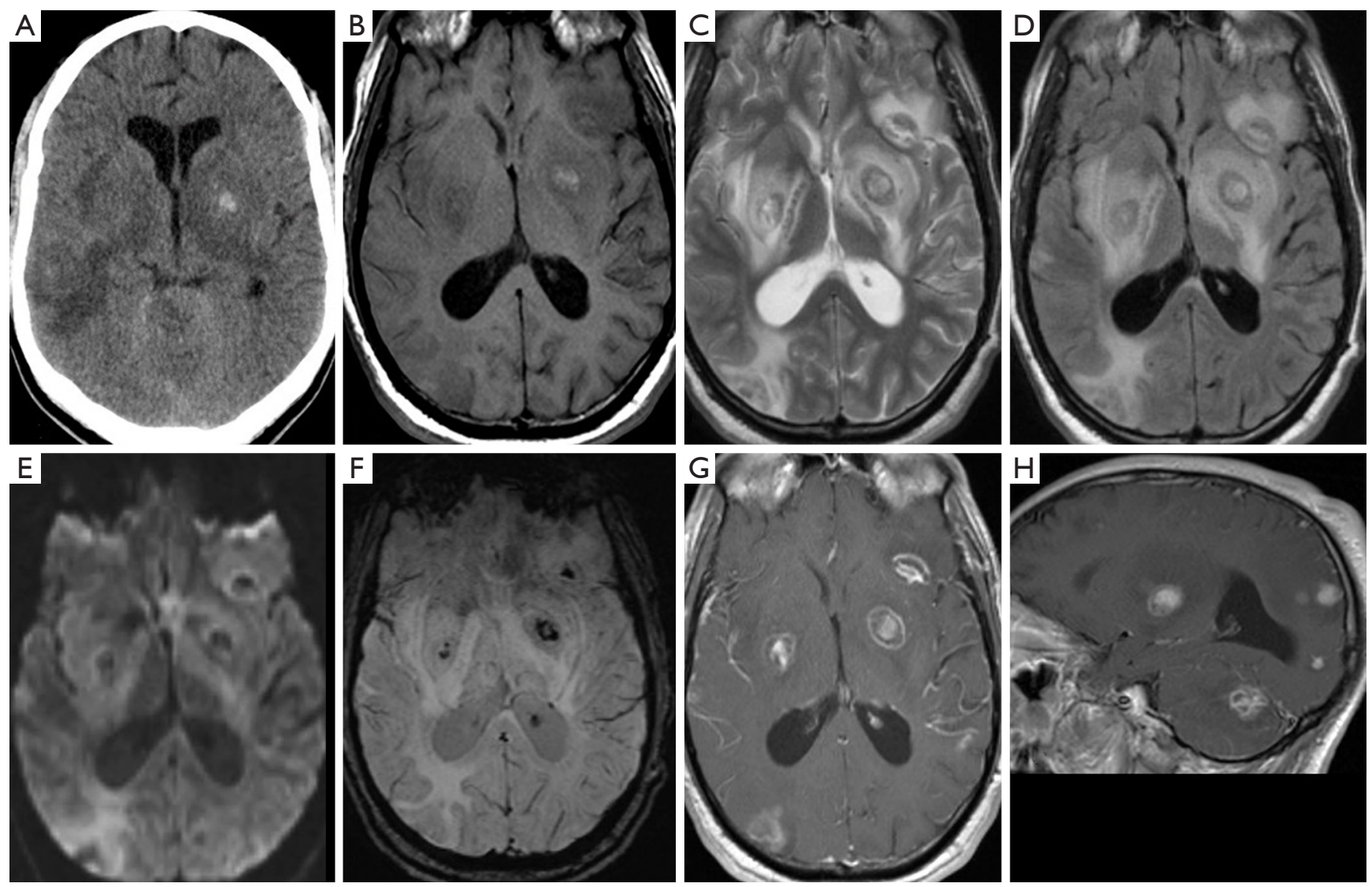

Figure 11 Toxoplasma encephalitis. A 38-year-old previously healthy male presented to ED with increasing somnolence, headaches, ataxia, dizziness and vomiting. During hospitalization, he was tested positive for HIV. His CD4 was 40 . Axial NECT image (A) showed multiple irregular hypodense areas of various sizes throughout the brain. One of the lesions in the left basal ganglia demonstrated a central hyperdense focus, which also appeared hyperintense on axial T1 image (B). This may represent a focal acute hemorrhage. The rest of the lesions appeared diffusely hypointense on axial T1 image. On Axial T2 and FLAIR (C,D) images, multiple small lobulated lesions with hypointense rim and hyperintense centers were noted, especially within bilateral basal ganglia. They demonstrated hyperintense rim and hypointense center on axial DWI (E). Susceptibility artifacts in these centers on axial GRE images (F) are consistent with focal hemorrhages. On post contrast axial and sag T1 images $(\mathrm{G}, \mathrm{H})$, they showed heterogeneous enhancement; Many of them have a "target' appearance. There was significant surrounding edema. Biopsy of right temporal lobe lesion showed "cerebral toxoplasmosis". (All images of this case are from the Hospitals of the University of Massachusetts School of Medicine at Baystate Health System.) ED, erectile dysfunction; HIV, human immunodeficiency virus; NECT, nonenhanced CT; FLAIR, fluid-attenuation inversion recovery; DWI, diffusion weighted images.

with sulfadiazine and folinic acid is the most common regime, typically for 6 weeks. Initial therapy is followed by secondary prophylaxis or maintenance therapy which is continued until CD4 counts are over 200 cells/microliters for more than three months.

\section{B. Cryptococcal meningoencephalitis (CME)}

Cryptococcus neoformans is a yeast-like fungus that may result in opportunistic infections in immunocompromised patients. It is the most common fungal infection in $\mathrm{HIV}$ positive patients and the AIDS-defining illness for $60-70 \%$ of HIV-positive patients. CNS cryptococcal infection is the third most common infections of the brain besides HIV encephalopathy and TE. It likely occurs in patients with recently diagnosed $\mathrm{HIV}$ infection and with CD4 counts $<100$ cells $/ \mu$. This infection is responsible for 600,000 deaths worldwide each year, predominantly in populations with less access to HAART therapy. Its incidence has significantly declined in recent years with the advent of 


\section{HAART $(53,54)$.}

The most common manifestation of CNS cryptococcal infection is meningoencephalitis (CME) as brain parenchyma is almost always involved, though sometimes only detected by histologic examination (55). The inflammatory response in the brain is generally milder than that seen in bacterial meningoencephalitis. Cryptococci tend to proliferate in the subarachnoid spaces and spread into the perivascular spaces. Growth of the microorganism leads to formation of mucoid materials in the perivascular spaces, commonly referred as gelatinous pseudocysts. They are usually seen in the basal ganglia, corticomedullary junction and dentate nuclei. In general, involvement of the brain is diffuse, but localized infections-cryptococcomas, may also be seen in about $10 \%$ of patients.

Symptoms of CME usually develop indolently over a period of weeks. They are nonspecific including headache, fever, malaise. Classical meningeal symptoms and signs, such as neck stiffness, photophobia and vomiting are present in $1 / 4$ to $1 / 3$ of patients. Isolated fever and headaches in advanced AIDS patients with CD $4<100$ cells/mL should raise a high index of suspicion for CME.

LP shows a decreased WBC count with a mononuclear predominance, slightly elevated protein and low glucose concentration in CSF; elevated opening pressure has been observed in $70 \%$ of AIDS patients but less frequent in nonHIV-infected patients. Diagnosis of CME is established by identification of cryptococcal yeast on Indian ink stain smear of CSF (75\%) or CSF culture; other highly sensitive and highly specific diagnostic tests in AIDS patients (greater than $90 \%$ sensitivity and specificity) are finding cryptococcal antigen $(\mathrm{CrAg})$ and positive cryptococcal PCR in $\operatorname{CSF}(53,54,56)$.

The challenge in diagnosing CNS cryptococcal infections lies in its wide spectrum of nonspecific neuroimaging findings, from normal to nonspecific patterns of meningitis, meningoencephalitis, and multiple abscesses. Typical findings of a subacute cryptococcal meningitis or meningoencephalitis include meningeal enhancement without or with abnormal signals of the adjacent brain. The meningeal enhancement is not commonly present on both CT and MR but tend to build along the brain base when present. A communicating hydrocephalus may raise clinical suspicion. Gelatinous pseudocysts on NECT show multiple small hypodense cysts in or around the basal ganglia and the corticomedullary junctions. On MRI, these cysts appear hypointense on T1 and hyperintense on T2/FLAIR but are slightly different than CSF signals. They may show no or mild peripheral enhancement. Perilesional edema is generally not evident. Restricted diffusion is usually not seen in most lesions, but its presence in the absence of meningeal enhancement or parenchymal enhancement should be a strong indicator of this disease. Cryptococcomas, either as a solid enhancing mass or as disseminated enhancing nodules in the midbrain and basal ganglia, are found in $4-11 \%$ cases. Multiple military enhancing parenchymal and leptomeningeal nodules with choroid plexus involvement are demonstrated in rare cases $(18,52,54)$ (Figure 12).

The standard antifungal therapy, Amphotericin B in combination with flucytosine, often performs poorly. Steroids do not improve survival and may contribute to adverse effects in cryptococcal meningitis, thus are only recommended in a subgroup of patients who have cryptococcomas with mass effect or IRIS $(53,54)$.

\section{Progressive multifocal leukoencephalopathy (PML)}

PML is a rare and fatal opportunistic infection, caused by the John Cunningham (JC) virus. It occurs in up to $5 \%$ of AIDS patients, especially when patient's CD4 counts is $<200$ cells $/ \mathrm{mL}$. People on chronic immunosuppressive medications are also at increased risk. Although the infection rate has gone down in the late-HAART era, poor prognosis still makes PML a daunting diagnosis. If untreated, the mortality rate is $30-50 \%$ within the first three months of diagnosis (57-59).

The JC virus is ubiquitously present in human bodies in dormant forms. Reactivation of the latent virus affects oligodendrocytes of the brain in immunocompromised patients leading to massive demyelination of white matter.

Clinical signs and symptoms are diverse, depending on affected locations of the brain and the degree of damage, typically including clumsiness, progressive weakness, visual defects (cortical blindness), language disturbance, behavioral changes, etc. Symptoms may evolve over the course of weeks to months, progressing rapidly in the last few months which usually leads to severe disability or frequently death (57-59).

Diagnosis of PML can be made on combination of positive CSF viral PCR of JC virus and progressive white matter disease on neuroimaging. Routine blood studies should include HIV serology and viral load if HIV status is unknown. PCR is the preferred way of diagnosis, but brain biopsy remains the gold standard when another disease entity such as CNS lymphoma is part of the differential 

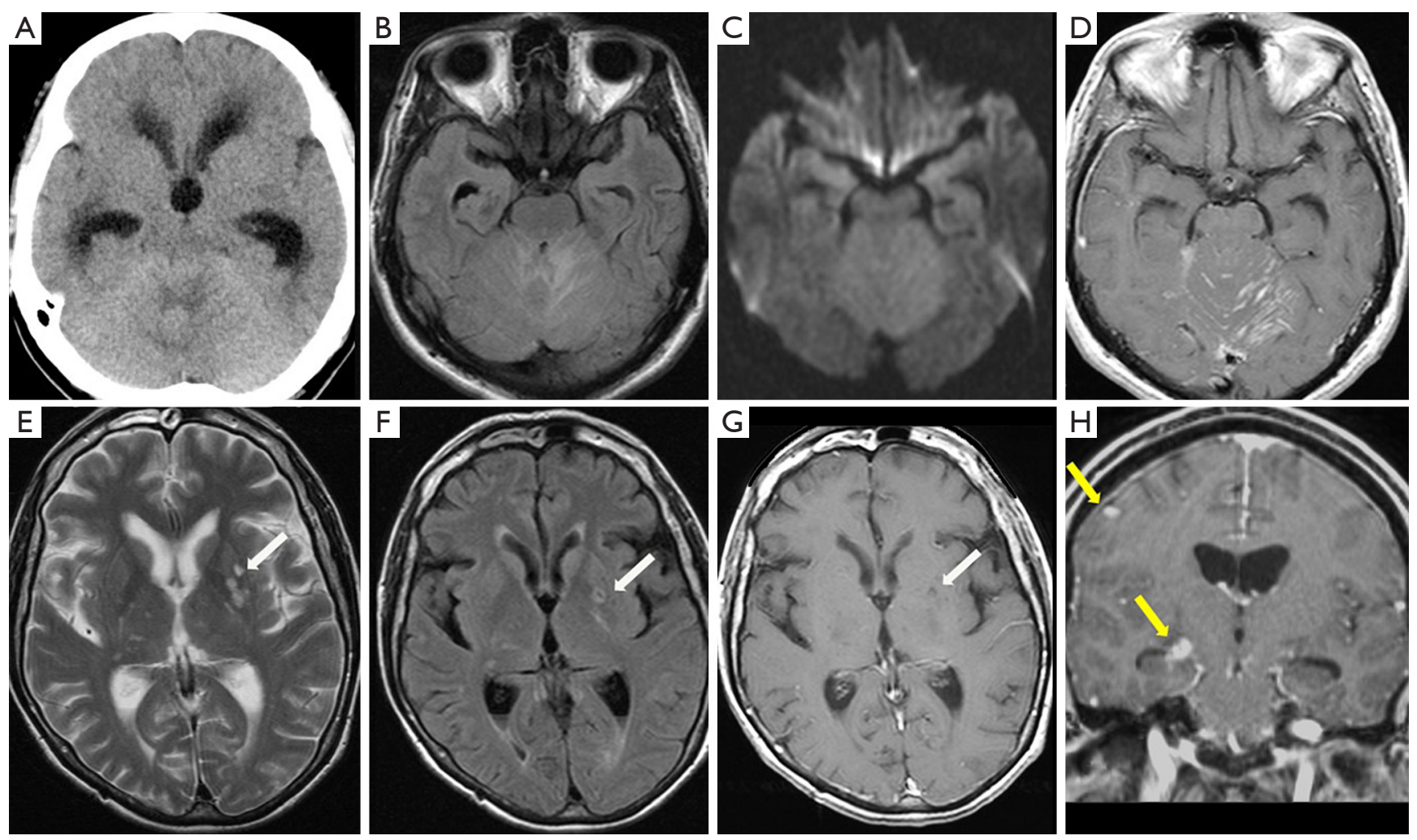

Figure 12 Cryptococcal Meningoencephalitis. A 30-year-old male with history of AIDS, presented with severe headaches, confusion, nausea and vomiting. He had been on and off HAART for recent years. Axial NECT (A) showed significant hydrocephalus. There were illdefined hypodense areas in the cerebellar white matters bilaterally with effacement of the sulci. These areas appeared hyperintense on axial FLAIR image (B). They showed no restricted diffusion on axial DWI (C). Post contrast axial T1 image (D) showed diffuse leptomeningeal enhancement along the sulci of the cerebellum bilaterally. The findings were consistent with meningoencephalitis. The patient's CSF had high titers of CrAg. In another patient with confirmed CNS cryptococcal infection, multiple small cysts were seen within the right thalamus and the left basal ganglia on axial T2 and FLAIR images (E,F), which show no enhancement on post contrast axial T1 image (G), suggesting "gelatinous pseudocysts". Solid enhancing nodules were also seen in the brain on post contrast coronal T1 image (H), consistent with "cryptococcoma". (All images of these cases are from the Hospitals of the University of Massachusetts School of Medicine at Baystate Health System.) AIDS, acquired immunodeficiency syndrome; HAART, highly active antiretroviral therapy; NECT, nonenhanced CT; DWI, diffusion weighted images; CSF, cerebral spinal fluid; FLAIR, fluid-attenuation inversion recovery.

diagnosis. Histopathologic triad of lytic demyelination of oligodendrocytes, bizarre astrocytes, and enlarged oligodendroglial nuclei are characteristic on brain biopsy or autopsy (58-60).

Typical white matter disease caused by PML consists of unilateral or bilateral but asymmetrical areas of demyelination that do not conform to any vascular territories in the brain. Mass effect is seen in acute phase, while encephalopathic changes with atrophy and volume loss are observed in chronic PML. Contrast enhancement is rare and, if it occurs, is patchy. The supratentorial lobar white matter is the most common location. PML lesions usually begin in the subcortical white matter of the parietooccipital or frontal lobes but may also involve the corpus callosum, brainstem, pyramidal tracts, and cerebellum. The second most common location is cerebellar peduncle. At the early stage, patients may show small, scattered subcortical white matter foci, which later develop bilateral asymmetric, large confluent lesions extending to the subcortical U fibers. The so-called "scalloping out" of the gray-white border refers to sparing the cortical ribbon. The cortex is generally spared even in advanced disease $(59,61,62)$.

CT of the head may reveal focal ill-defined hypodensities in subcortical and periventricular white matters without 

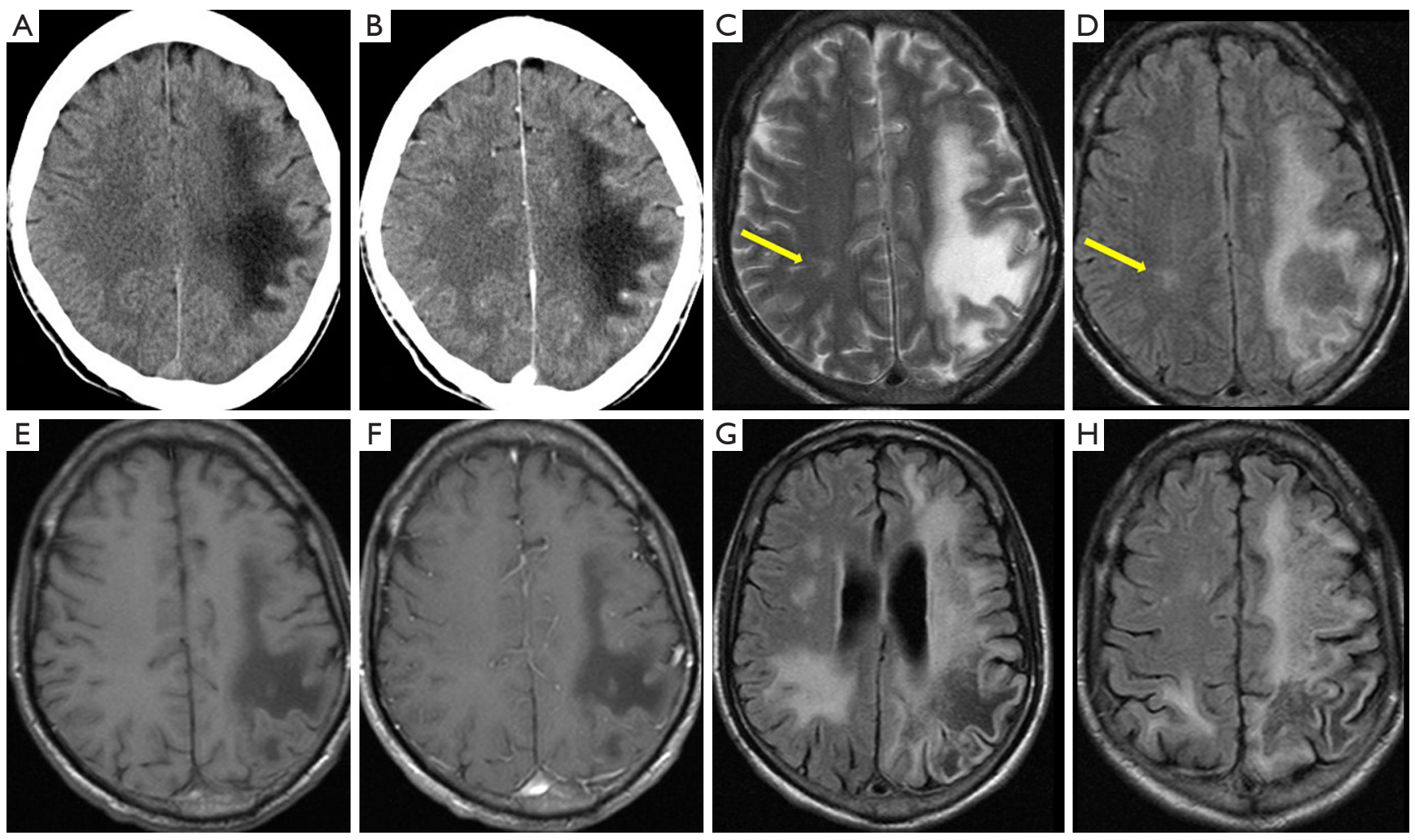

Figure 13 Progressive multifocal leukoencephalopathy (PML). A 49-year-old male with AIDS but noncompliant of HAART regimen, presented with right facial hemiparesis, right upper and lower extremity weakness, and slurred speech. Axial NECT and CECT images (A,B) showed a large irregular area of hypodensity within the white matter of the left frontal and parietal lobes without enhancement. On MR, axial T2 and FLAIR image (C,D) showed a similar area of hyperintense T2 and hypointense FLAIR signals in the left parietal white matter with peripheral hyperintense T2 and FLAIR signals which extended to left frontal white matter. A small hyperintense T2/FLAIR focus was seen in the right parietal white matter (arrow). Pre-and postcontrast T1 images (E,F) showed no enhancement of these lesions. PML was diagnosed by CSF testing. Subsequently, the abnormal signals in the brain showed significant progression within the whiter matters of bilateral cerebral hemispheres on axial FLAIR images $(\mathrm{G}, \mathrm{H})$ despite clinical improvement of the patient's symptoms. The findings were consistent with IRIS. (All images of this case are from the Hospitals of the University of Massachusetts School of Medicine at Baystate Health System.) AIDS, acquired immunodeficiency syndrome; HAART, highly active antiretroviral therapy; NECT, nonenhanced CT; CECT, contrast-enhanced computed tomography; FLAIR, fluid-attenuation inversion recovery; CSF, cerebral spinal fluid; IRIS, immune reconstitution inflammatory syndrome.

mass effect and little contrast enhancement. These lesions could be unilateral and small at the beginning but invariably progress to a more confluent appearance with bilateral but asymmetrical distribution in the white matters. On MR, these lesions appear hypointense on T1 and hyperintense on T2/FLAIR images. "Spongy" lesions with marked hyperintense foci within the background of relatively less hyperintense confluence are found in later stages of PML. On DWI, PML lesions may show a hyperintense rim along the periphery or advancing edge of the lesion but no or little hypointense signals on corresponding ADC images $(61,62)$.
Enhancement is not typically seen in HIV-related PML without IRIS $(18,63,64)$. IRIS is a syndrome of paradoxical worsening of neuroimaging findings when patients' clinical symptoms improve while receiving HAART therapy. Interestingly, lesion enhancement on CT or MRI is found to be a predictor of long-term survival in AIDS patients with PML in one study $(62,63)$ (Figure 13).

Major differential diagnoses of PML include posterior reversible encephalopathy syndrome (PRES) in non-immunocompromised patients and lymphoma in immunodeficient patients. PRES can be caused by 
malignant hypertension, eclampsia and some medical treatments. Typical finding is relative symmetrical edema of subcortical white matters, especially on both sides of the parietal and occipital lobes. CT images show illdefined hypodensities of the affected areas without mass effect. On MRI, these lesions show hypointense T1 and hyperintense T2 signals without enhancement. Because these lesions represent vasogenic cerebral edema, DWI shows iso- or hypo-intense signals with hyperintense signals on ADC map (65). CNS lymphoma may mimic PML lesions on non-enhancement images. But it often shows cortical involvement, moderate edema and diffuse contrast enhancement as well as restricted diffusion. CSF analysis is also helpful for its differentiation.

There is no effective treatment PML, besides restoring the immune system with HAART. Paradoxical brain damage resulting in fulminant IRIS following HAART further complicates the PML course, subsequently requiring early and long-term steroid therapy. Prognosis is poor even with treatment. Studies have shown a 6-month mortality of $45.5 \%$ in late-presenting HIV-positive patients with PML, and a 1 -year survival of $46.4 \%$ in patients on HAART.

\section{Spinal osteomyelitis (OM)/discitis and spinal epidural abscess (SEA)}

$\mathrm{OM}$ and discitis of the spine are bacterial infections of the vertebral bodies and the intervertebral discs. SEA is an infectious fluid collection (pus) within the epidural spaces of the spinal canal. SEA is usually concomitant with spinal $\mathrm{OM}$ and discitis, although at early stages, each of them can exist independently.

Native vertebral OM/discitis cases are uncommon, with an estimated incidence of 4.8 cases per population of 100,000 . The incidences have doubled from 1998 to 2013 (66). It is a serious condition with a reported 1-year mortality rate of $11 \%$. OM primarily occurs in adults, with half of patients are more than 59 years old. Male (51\%) and female are almost equally affected. However younger people have become more frequently affected in the United States due to the IV drug abuse epidemic (67).

Staphylococcus aureus is the most common isolated organism in native $\mathrm{OM}$ or $\mathrm{OM}$ after previous instrumentation (indwelling catheter, spinal surgery) as well as SEA, accounting for $1 / 2$ to $2 / 3$ of cases in developed countries. Coagulate-negative Staphylococcus and Streptococcus are second most common in native $\mathrm{OM}$ and $\mathrm{OM}$ after instrumentation respectively (68). Other etiologies include Gram-negative or anaerobic organisms. OM in IV drug abusers had a higher prevalence of polymicrobial infection (69). Identification of the inciting bacteria could be done by CT guided biopsy and open surgery biopsy which, when combined, showed more yield than blood cultures ( $77 \%$ vs. 58\%).

The most common route of transmission of OM/ discitis is hematogenous spread from other part of body, such as urinary tract infection or endocarditis. Contiguous spread from the infected adjacent structures such as aorta, esophagus can be seen, and many times accompanied by paravertebral phlegmon or abscesses. Previous trauma or instrumentation (i.e., spinal surgery) increases the risks of the infection. IV drug abuser and previous instrumentation have higher incidence of methicillin-resistant Staphylococcus aureus (MRSA) infection. The rate of recurrent infection is also higher among MRSA infections. Higher mortality rate is associated with increased age, male gender, and other related clinical conditions such as congestive heart failure, cerebrovascular diseases, liver or renal diseases (70-72).

The lumber spine is most commonly affected, followed by thoracic spine and cervical spine. Since segmental arteries supplying the vertebrae usually bifurcate to supply two adjacent end plates of contiguous vertebrae, thus hematogenous vertebral OM often causes bone destruction in two adjacent vertebral bodies and usually destroys their intervertebral disc. Posterior extension of $\mathrm{OM} /$ discitis causes epidural phlegmon or abscess. The facet joints and the posterior elements may be involved. When the facet joint is involved, it is called septic arthritis of the facet joint, which nowadays becomes facetitis, a newly invented medical term.

The most common presentation of $\mathrm{OM} /$ discitis is back pain, localized at the affected area of spine. Back pain usually begins insidiously and progressively worsens over several weeks to months. At the beginning, the pain may be worse at night and relieved by bed rest. Fever may be seen in the affected individual but not a consistent presentation. Neurologic deficits are more common with accompanied epidural abscess. Definitive diagnosis of OM/discitis or epidural SEA is made by positive culture from the biopsy specimen of the infected sites. Back pain, fever and classic radiographic finings of $\mathrm{OM} /$ discitis are sufficient to start antimicrobial therapy for suspected cases. Positive blood culture significantly increased the index of suspicion. LP is usually not helpful and may increase the risk of the pathogen entering the thecal sac. Laboratory studies of the blood are nonspecific and often show leukocytosis, elevated erythrocyte sedimentation rate (ESR) and positive C-reactive protein (CRP) (69-72). 


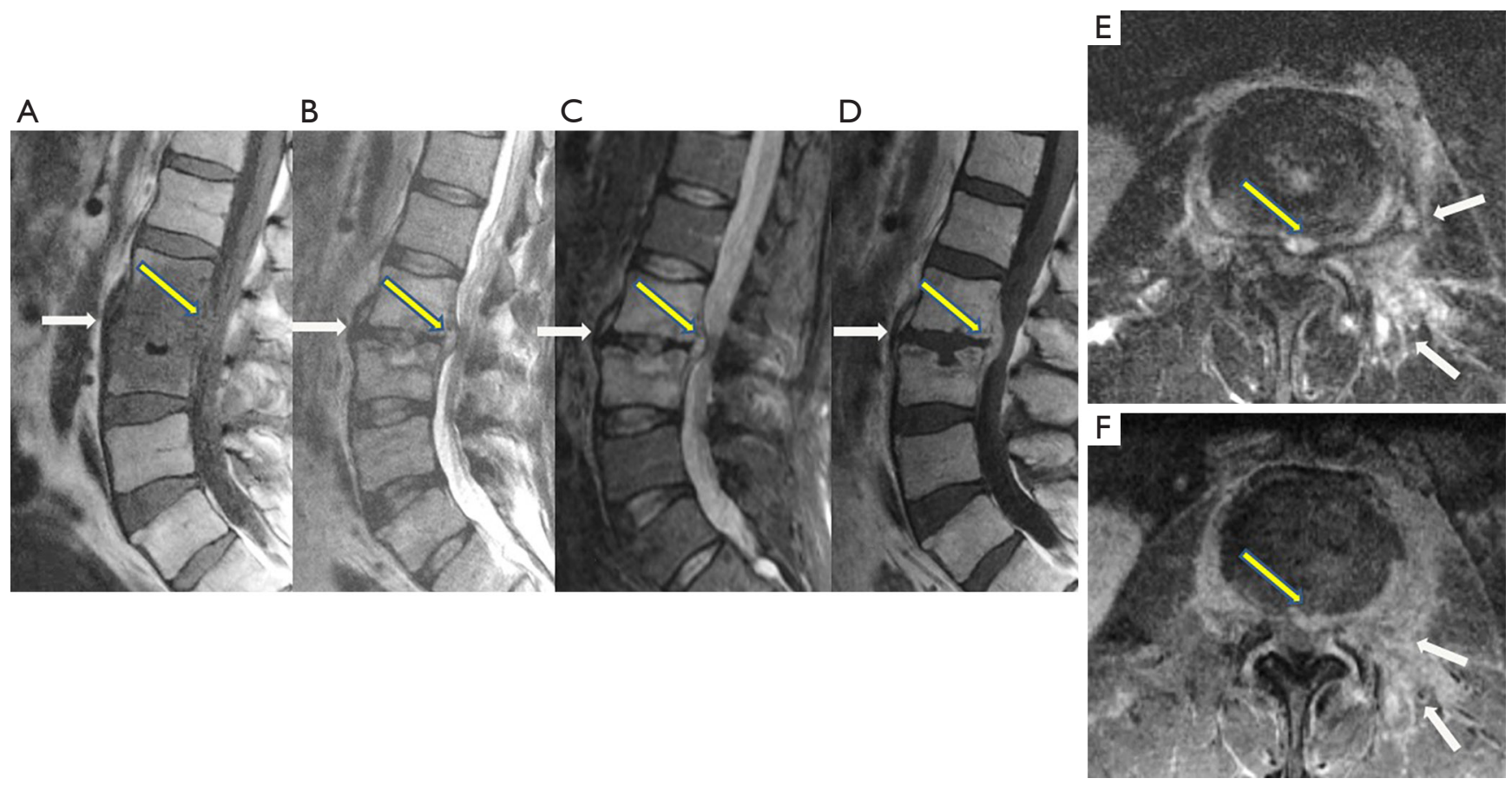

Figure 14 Spinal osteomyelitis and discitis. A 50-year-old man with history of diabetes and polysubstance abuse, presented with low back pain, radiating down to his legs. Urgent lumbar MR demonstrated hypointense T1 signals involving L2 and L3 vertebral bodies and the L2-3 intervertebral disc on sag T1 image (A), and heterogeneously hyperintense signals on sag T2 and STIR images (B,C). These areas showed diffuse enhancement on postcontrast sag T1 image (D). The inferior endplate of L2 and the superior endplate of L3 as well as L2-3 intervertebral disc appeared diffusely irregular suggesting erosions. The findings were consistent with acute osteomyelitis and discitis. L3 vertebral body had mildly decreased vertebral body height, consistent with a pathological compression fracture. There was a small amount of prominent soft tissues with the ventral epidural space posterior to L2 and L3 vertebral bodies, centered at the L2-3 intervertebral disc space (yellow arrows). These soft tissues were enhancing heterogeneously, consistent with epidural phlegmon. Prominent soft tissues were also seen surrounding the vertebral bodies extending to adjacent psaos muscles and paraspinal muscles (white arrows), which appeared heterogeneously hyperintense on axial T2 with fat -sat image (E) and showed diffuse enhancement on post contrast axial T1 (F) with fat-sat image, consistent with paravertebral and paraspinal phlegmon. They may contain a few small walled-off abscesses. CT-guided biopsy of the lumbar spine showed Gram-negative rods which were identified as Citrobacter freundii. (All images of this case are from the Hospitals of the University of Massachusetts School of Medicine at Baystate Health System.)

Plain radiography of the spine is insensitive for detecting $\mathrm{OM} /$ discitis until late stages of the disease. Permeated osteolytic area within the vertebral body, cortical irregularities and intervertebral disc destruction can be seen in the advanced stage, but earlier and more easily identified by CT. Contrast enhancement is of limited value for bony or disc destructions but may delineate the associated epidural empyema, phlegmon or abscesses within the adjacent paravertebral spaces and/or paraspinal muscles. On MR, the bone marrow or disc edema are early findings of $\mathrm{OM} /$ discitis, which demonstrate hypointense T1 and hyperintense T2/ STIR signals as well as diffuse enhancement after intravenous contrast. Frank or advanced $\mathrm{OM}$ and discitis demonstrate bony destructions or disc irregularities of the infected bony structures or discs. The corresponding hyperintense T2 and STIR signals as well as enhancement become more heterogeneous (Figure 14). Accompanied phlegmon or abscess may be identified in the related epidural space as well as the adjacent perivertebral and paraspinal spaces or muscles. In later stage of the disease, the vertebral body may show a pathological fracture (70-72).

SEA is one of the most frequent causes for urgent MRI in the Emergency Department, especially with the clinical triad of back pain, fever, and neurological deficits involving the extremities. It is considered a neurological emergency as its complication of cord compression or infarct may cause 

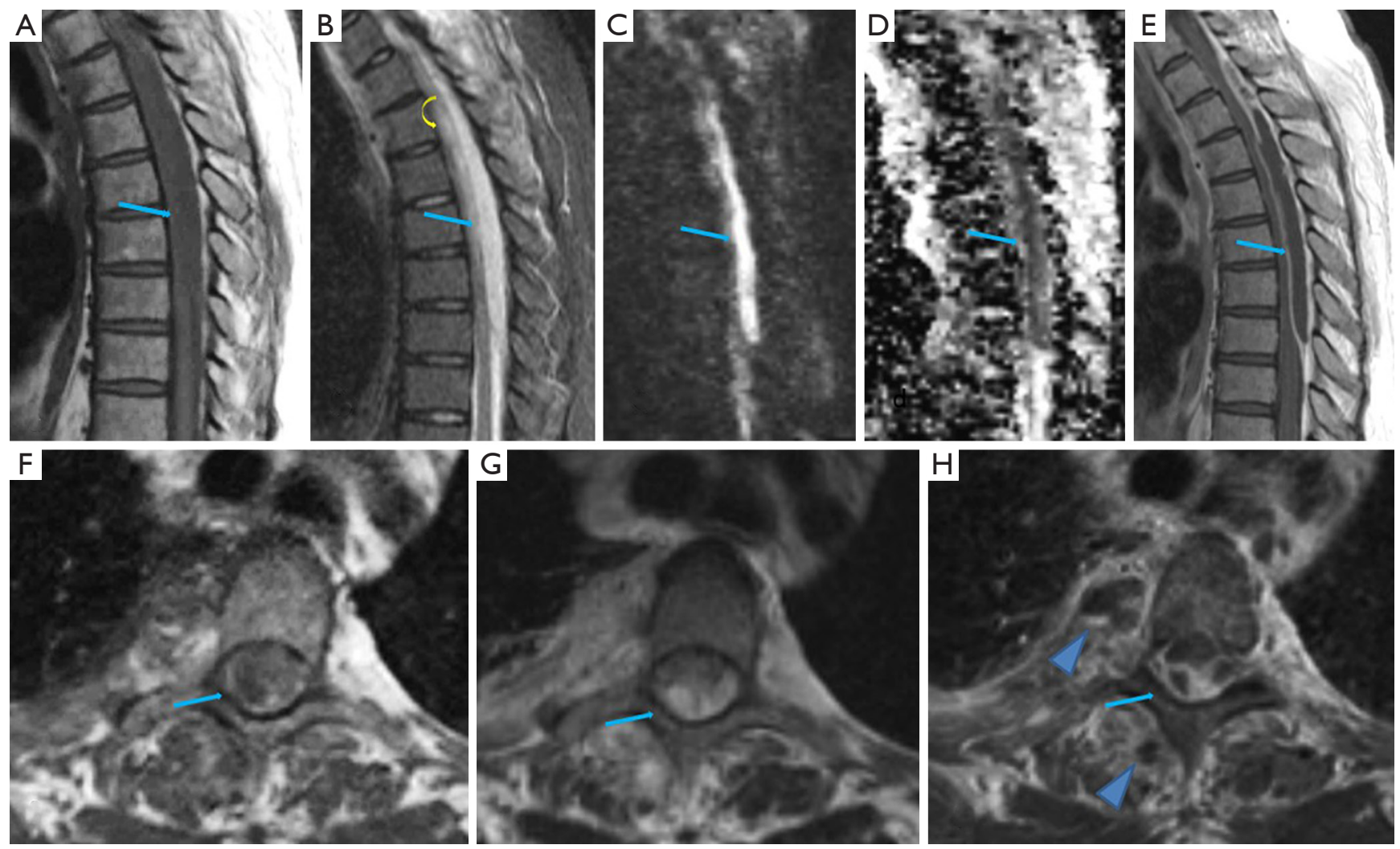

Figure 15 Spinal epidural abscess (SEA). A 50-year-old female presented with right shoulder pain, upper back pain, mild numbness and tingling sensation. She had no fever. ESR was normal. On MR, there was an elongated fluid collection (arrows) within the posterior epidural space compressing the cord and displaced it anteriorly on sag T1, T2 (A,B) and axial T1, T2 images (F,G). Hyperintense T2 foci were noted within the cord suggesting cord edema (curved arrow). Sag DWI and ADC images (C,D) demonstrated restricted diffusion of this epidural fluid collection, which showed diffuse rim enhancement on post contrast sag and axial T1 image (E,H). This was consistent with a multiloculated epidural abscess. There were also multiple fluid collections of different sizes with peripheral enhancement within the paravertebral and paraspinal spaces, consistent with abscesses (arrow heads). Culture of the drainage from the laminectomy grew MRSA. (All images of this case are from the Hospitals of the University of Massachusetts School of Medicine at Baystate Health System.) ESR, erythrocyte sedimentation rate; DWI, diffusion weighted images; ADC, apparent diffusion coefficient.

irreversible neurological deficits, paralysis or even death. It has similar epidemiology as $\mathrm{OM} /$ discitis. In addition to the hematogenous seeding from septicemia into the epidural space, the spread of bacterial infection can also arise from the direct invasion from adjacent infected structures such as joint or disc spaces.

Although CT may reveal evidence of discitis or OM if present, SEA commonly escapes detection. MRI with contrast is the only true modality that can clearly diagnose SEA. Identification of an epidural fluid collection with rim-enhancement is generally sufficient for a presumptive diagnosis of SEA in the right clinical setting. The disease could present as either a phlegmonous or liquid form. Phlegmonous forms will demonstrate prominent heterogeneously enhancing soft tissues while a liquid form will show a single or multiple fluid collections with peripheral rim-like enhancement (73). Longitudinal extension is common with average extent ranging from 3 to 5 spinal cord segments (74). Characteristically, restricted diffusion of the epidural fluid collection is the hallmark of epidural abscess and separate if from other types of epidural fluid collections, such as a hematoma or an arachnoid cyst. But DWI images of spine are not usually acquired in most institutions (75). Additionally, SEA is often associated with inflammatory reaction or infection of the adjacent paraspinal muscles, psoas muscles and facet joints (Figure 15).

Differential diagnosis of $\mathrm{OM} /$ discitis include degenerative disease and osteolytic metastasis. Although 
back pain is also common in degenerative disease of spine, but the patients' clinical history is usually different and the features of infection such as fever, is not typically present. Bony or disc destructions, and pathological fractures do not exist. Spinal metastasis, beside distinct history of a primary tumor elsewhere, often demonstrates vertebral body destructions and can be associated with a pathological fracture. But the intervertebral disc is typically spared as the avascular disc is resistant to tumor invasion (76). The associated epidural metastasis appears a solid enhancing mass focused on the level of bony involvement rather than an elongated epidural fluid collection with peripheral enhancement of SEA. Another major differential diagnosis of SEA is an acute epidural hematoma of the spine. Beyond different clinical history, key features to distinguish SEA from epidural hematoma are mostly based on signal characteristics on MR. The signals of the epidural hematoma appear similar to acute hematoma intracranially. They change with time due to evolution of the blood products. The acute epidural hematoma should not demonstrate peripheral enhancement (77). Spinal epidural metastases can also mimic SEA, though their clinical courses are usually more chronic and not associated with clinical history of fever, leukocytosis, or elevated ESR.

Treatment of $\mathrm{OM} /$ discitis usually begins with intravenous therapy by broad-spectrum empiric antibiotics for patients with sepsis or neurological comprises. When the causative microorganism is known, antimicrobial therapy should be tailored to the identified pathogen. Vancomycin is reserved for MRSA infection, commonly seen in IV drug abusers.

Surgical decompression is the most common treatment method for SEA, followed by IV antimicrobial therapy.

\section{Conclusions}

Infectious diseases of the CNS usually have very high morbidity and mortality. Despite advances in medicine, accurate and prompt diagnosis of these infections are still very challenging due to the poor specificity of the patients' presenting signs and symptoms, especially in pediatric, elderly, and immunocompromised populations. Objective findings on neuroimaging studies play a critical role in pinpointing the exact diagnoses so that treatments can be initiated in a timely fashion. Since prompt treatment is paramount for the patients' condition and may even be lifesaving, it is pivotal for clinicians to become familiar with the features of a wide spectrum of the CNS infections, their imaging patterns and characteristics as well as differential diagnoses. A pattern approach method of these diseases can also be developed. As a result, the patients can have a significantly improved prognosis with reduced morbidity and mortality.

Tables 1,2 list the major clinical features and imaging features of the common infectious diseases of the CNS

Table 1 CT \& MR findings of the common infectious diseases of the CNS

\begin{tabular}{lll}
\hline \multirow{2}{*}{ Disease entities } & \multicolumn{1}{c}{ CT } & Imaging findings \\
\cline { 2 - 3 } $\begin{array}{l}\text { Acute meningitis/ } \\
\text { encephalitis }\end{array}$ & $\begin{array}{l}\text { Mild sulci effacement, hypo- or iso-dense } \\
\text { exudate, hydrocephalus (+/-), meningeal } \\
\text { enhancement }\end{array}$ & $\begin{array}{l}\text { Isointense T1 \& hyperintense FLAIR exudate, lepto- or } \\
\text { pachy-meningeal enhancement. Restricted diffusion of the } \\
\text { infected meninges, exudates \& the related encephalitis }\end{array}$ \\
$\begin{array}{l}\text { Herpes simplex } \\
\text { encephalitis type I } \\
\text { (HSV-1) }\end{array}$ & $\begin{array}{l}\text { Hypodensities in temporal lobe with } \\
\text { significant edema, focal hemorrhages, } \\
\text { necrosis, \& heterogenous enhancement }\end{array}$ & $\begin{array}{l}\text { Hypointense T1 \& heterogeneously hyperintense T2/FLAIR } \\
\text { signals in temporal lobe; focal hemorrhages \& necrosis; patchy } \\
\text { enhancement; heterogeneous \& variable DWI signals which may } \\
\text { be seen earlier than abnormalities on T2/FLAIR }\end{array}$ \\
$\begin{array}{l}\text { Herpes simplex } \\
\text { (HSV-2) }\end{array}$ & $\begin{array}{l}\text { Multiple ill-defined hypodensities of various } \\
\text { sizes and locations, loss of gray-white } \\
\text { matter junctions, cerebral edema }\end{array}$ & $\begin{array}{l}\text { Multifocal areas of hypointense T1 \& hyperintense T2/FLAIR } \\
\text { signals, loss of gray-white matter junction, focal hemorrhages, } \\
\text { variable restricted diffusion in cortex and white matters, \& various } \\
\text { meningeal or parenchymal enhancement }\end{array}$ \\
$\begin{array}{l}\text { Eastern equine } \\
\text { encephalitis (EEE) }\end{array}$ & $\begin{array}{l}\text { Diffuse heterogeneous hypointense areas } \\
\text { within bilateral basal ganglia \& thalami, } \\
\text { associated with significant swelling and } \\
\text { mass effect. No hemorrhage }\end{array}$ & $\begin{array}{l}\text { Heterogeneously hypointense T1 \& hyperintense T2/FLAIR signals } \\
\text { in basal ganglia \& thalami bilaterally with significant swelling; No } \\
\text { restricted diffusion or enhancement; Variable cortical \& brainstem } \\
\text { involvement }\end{array}$ \\
\hline
\end{tabular}

Table 1 (continued) 
Table 1 (continued)

\begin{tabular}{|c|c|c|}
\hline Disease entities & \multicolumn{2}{|r|}{ Imaging findings } \\
\hline CNS tuberculosis (TB) & $\begin{array}{l}\text { TB meningitis: iso-or hyperdense basilar } \\
\text { exudates, hydrocephalus. Uniform and } \\
\text { intense enhancement along basilar } \\
\text { meninges. Tuberculoma: hypodense lesion } \\
\text { significant edema }\end{array}$ & $\begin{array}{l}\text { TB meningitis: hypo- or iso-intense T1 \& hyperintense T2/FLAIR } \\
\text { exudates w. intense enhancement along basilar meninges. } \\
\text { Tuberculomas: discrete hypointense T1 \& hyperintense T2 nodules } \\
\text { w. ring or homogeneous disc-shaped enhancement; Restricted } \\
\text { diffusion in necrotic centers of larger tuberculomas }\end{array}$ \\
\hline $\begin{array}{l}\text { Pyogenic brain } \\
\text { abscess (BA) }\end{array}$ & $\begin{array}{l}\text { A hypodense cavitating lesion w. } \\
\text { dependent debris \& thick or nodular rim } \\
\text { enhancement; surrounded by edema \& } \\
\text { mass effect }\end{array}$ & $\begin{array}{l}\text { A cavitating lesion w. hypointense T1 \& hyperintense T2/FLAIR } \\
\text { center which shows marked restricted diffusion; The surrounding } \\
\text { capsule shows a hypointense or intermediate rim on T2/FLAIR } \\
\text { images and diffuse ring enhancement }\end{array}$ \\
\hline $\begin{array}{l}\text { Septic embolic } \\
\text { encephalitis (SEE) }\end{array}$ & $\begin{array}{l}\text { Multiple small hypodense foci } \\
\text { w or w/o microbleeds; w. rim or } \\
\text { punctate enhancement, mostly at the } \\
\text { corticomedullary junctions }\end{array}$ & $\begin{array}{l}\text { Multiple small abscesses of different ages w. central restricted } \\
\text { diffusion at the watershed areas or gray-white matter junctions; w } \\
\text { or w/o microbleeds; Focal ischemic infarcts in MCA distribution; } \\
\text { Mycotic aneurysm seen in } 10 \% \text { cases }\end{array}$ \\
\hline $\begin{array}{l}\text { Toxoplasma } \\
\text { encephalitis (TE) }\end{array}$ & $\begin{array}{l}\text { Multiple hypodense lesions with significant } \\
\text { vasogenic edema, with a predilection for } \\
\text { the basal ganglia and thalami. Thin smooth } \\
\text { rim enhancement or eccentric nodular } \\
\text { enhancement }\end{array}$ & $\begin{array}{l}\text { Multiple focal hypointense T1 and hyperintense or mixed T2 } \\
\text { lesions with ring enhancement in similar locations; No restricted } \\
\text { diffusion; Significant perilesional edema; "Target Sign" in some } \\
\text { cases }\end{array}$ \\
\hline $\begin{array}{l}\text { Spinal osteomyelitis } \\
\text { (OM) \& discitis and } \\
\text { spinal epidural abscess } \\
\text { (SEA) }\end{array}$ & $\begin{array}{l}\text { Permeated osteolysis of vertebral bodies } \\
\text { and destruction disc spaces, paravertebral } \\
\text { soft tissue swelling, pathological fractures } \\
\text { at later stage; Epidural fluid collection } \\
\text { may be seen in SEA, especially with rim } \\
\text { enhancement }\end{array}$ & $\begin{array}{l}\text { Bone marrow edema \& homogeneous enhancement at early } \\
\text { stage of OM, more heterogeneous w. bony or disc destructions \& } \\
\text { pathological fractures at later stages; SEA appears as an epidural } \\
\text { fluid collection w. restricted diffusion \& diffuse rim-enhancement; } \\
\text { Abscesses can be seen in adjacent perivertebal \& paraspinal } \\
\text { spaces or muscles }\end{array}$ \\
\hline
\end{tabular}


Table 2 CSF \& serology tests of the common infectious diseases of the CNS

\begin{tabular}{|c|c|c|}
\hline Disease entities & CSF analysis (lumbar puncture) & Serology tests \& others \\
\hline $\begin{array}{l}\text { Herpes simplex encephalitis } \\
\text { type II (HSV-2) }\end{array}$ & $\begin{array}{l}\text { (+) PCR of HSV-2; CSF analysis: mononuclear cell } \\
\text { pleocytosis, normal or moderately } \downarrow \text { glucose \& mildly } \\
\uparrow \text { protein }\end{array}$ & $\begin{array}{l}\text { EEG shows multifocal periodic epileptic form } \\
\text { discharges }\end{array}$ \\
\hline $\begin{array}{l}\text { Eastern equine encephalitis } \\
\text { (EEE) }\end{array}$ & $\begin{array}{l}\text { (+) anti-EEE IgM antibody; neutrophilic pleocytosis in } \\
\text { CSF }\end{array}$ & 4 folds' $\uparrow$ of anti-EEE antibody titers \\
\hline CNS tuberculosis (TB) & $\begin{array}{l}\text { (+) AFB stain or culture of TB bacillus; CSF analysis: } \\
\text { lymphocytic pleocytosis, } \downarrow \text { glucose and } \uparrow \text { protein }\end{array}$ & $\begin{array}{l}\text { Nonspecific blood analysis; TB at extra- } \\
\text { neural sites a strong indicator of CNS TB }\end{array}$ \\
\hline Ventriculitis/ependymitis & $\begin{array}{l}\text { Increase ICP; (+) gram stain or culture; leukocytosis, } \downarrow \\
\text { glucose \& } \uparrow \text { protein. }\end{array}$ & Nonspecific blood analysis \\
\hline $\begin{array}{l}\text { Septic embolic encephalitis } \\
\text { (SEE) }\end{array}$ & Lumbar puncture is not helpful & $\begin{array}{l}\text { (+) blood culture or gram stain; Nonspecific } \\
\text { blood analysis: leukocytosis, elevated ESR }\end{array}$ \\
\hline Toxoplasma encephalitis (TE) & $\begin{array}{l}\text { (+) PCR o for DNA of Toxoplasma Gondii; Brain biopsy } \\
\text { in difficult cases }\end{array}$ & $\begin{array}{l}\text { (+) anti-toxoplasma lgG antibodies; Serology } \\
\text { of HIV infection and viral load; Routine blood } \\
\text { tests including CD4 count }\end{array}$ \\
\hline
\end{tabular}

mentioned in this article.

\section{Acknowledgments}

I would like to express special thanks to Christopher M. Lin who provided valuable tips of formatting various document, figures, and tables using Microsoft Office Suite, and proofread this manuscript for every revision. I would also like to thank my colleague Dr. Tara M. Catanzano for her continuous encouragement and support for my academic endeavors.

Funding: None.

\section{Footnote}

Provenance and Peer Review: This article was commissioned by the editorial office, Quantitative Imaging in Medicine and Surgery. The article did not undergo external peer review.

Conflicts of Interest: All authors have completed the ICMJE uniform disclosure form (available at http://dx.doi. org/10.21037/qims-20-886). The authors have no conflicts of interest to declare.

Open Access Statement: This is an Open Access article 
distributed in accordance with the Creative Commons Attribution-NonCommercial-NoDerivs 4.0 International License (CC BY-NC-ND 4.0), which permits the noncommercial replication and distribution of the article with the strict proviso that no changes or edits are made and the original work is properly cited (including links to both the formal publication through the relevant DOI and the license). See: https://creativecommons.org/licenses/by-nc-nd/4.0/.

\section{References}

1. Thigpen MC, Whitney CG, Messonnier NE, Zell ER, Lynfield R, Hadler JL, Harrison LH, Farley MM, Reingold A, Bennett NM, Craig AS, Schaffner W, Thomas A, Lewis MM, Scallan E, Schuchat A; Emerging Infections Programs Network. Bacterial meningitis in the United States, 1998-2007. N Engl J Med 2011;364:2016-25.

2. Oordt-Speets AM, Bolijn R, van Hoorn RC, Bhavsar A, Kyaw MH. Global etiology of bacterial meningitis: A systematic review and meta-analysis. PLoS One 2018;13:e0198772.

3. Prober CG, Srinivas NS, Mathew R. Nelson Textbook of Pediatrics. Chapter 603, 2936-2948.e2.

4. Sáez-Llorens X, McCracken GH Jr. Bacterial meningitis in children. Lancet 2003;361:2139-48.

5. Mandell GL, Bennett JE, Dolin R. Mandell, Douglas, and Bennett's Principles and Practice of Infectious Diseases. 7th edition. Philadelphia, PA: Churchill Livingstone/ Elsevier, 2010.

6. van de Beek D, de Gans J, Spanjaard L, Weisfelt M, Reitsma JB, Vermeulen M. Clinical features and prognostic factors in adults with bacterial meningitis. N Engl J Med 2004;351:1849-59.

7. Troendle M, Pettigrew A. A systematic review of cases of meningitis in the absence of cerebrospinal fluid pleocytosis on lumbar puncture. BMC Infect Dis 2019;19:692.

8. Dorsett M, Liang SY. Diagnosis and Treatment of Central Nervous System Infections in the Emergency Department. Emerg Med Clin North Am 2016;34:917-42.

9. Hazany S, Go JL, Law M. Magnetic resonance imaging of infectious meningitis and ventriculitis in adults. Top Magn Reson Imaging 2014;23:315-25.

10. Mohan S, Jain KK, Arabi M, Shah GV. Imaging of meningitis and ventriculitis. Neuroimaging Clin N Am 2012;22:557-83.

11. Smirniotopoulos JG, Murphy FM, Rushing EJ, Rees JH, Schroeder JW. Patterns of contrast enhancement in the brain and meninges. Radiographics 2007;27:525-51.
12. Tosaka M, Sato N, Fujimaki H, Tanaka Y, Kagoshima K, Takahashi A, Saito N, Yoshimoto Y. Diffuse pachymeningeal hyperintensity and subdural effusion/ hematoma detected by fluid-attenuated inversion recovery MR imaging in patients with spontaneous intracranial hypotension. AJNR Am J Neuroradiol 2008;29:1164-70.

13. James SH, Kimberlin DW. Neonatal Herpes Simplex Virus Infection. Infect Dis Clin North Am 2015;29:391-400.

14. Whitley RJ. Herpes Simplex Virus Infections of the Central Nervous System. Continuum (Minneap Minn) 2015;21:1704-13.

15. Renard D, Nerrant E, Lechiche C. DWI and FLAIR imaging in herpes simplex encephalitis: a comparative and topographical analysis. J Neurol 2015;262:2101-5.

16. Fa F, Laup L, Mandelbrot L, Sibiude J, Picone O. Fetal and neonatal abnormalities due to congenital herpes simplex virus infection: a literature review. Prenat Diagn 2020;40:408-14.

17. Corey L, Whitley RJ, Stone EF, Mohan K. Difference between herpes simplex virus type 1 and type 2 neonatal encephalitis in neurological outcome. Lancet 1988;1:1-4.

18. Osborn AG, Hedlund GL, Salzman KL. Osborn's brain: imaging, pathology, and anatomy. Salt Lake City: Amirsys, 2013.

19. Lindsey NP, Staples JE, Fischer M. Eastern Equine Encephalitis Virus in the United States, 2003-2016. Am J Trop Med Hyg 2018;98:1472-7.

20. Armstrong PM, Andreadis TG. Eastern equine encephalitis virus--old enemy, new threat. N Engl J Med 2013;368:1670-3.

21. Deresiewicz RL, Thaler SJ, Hsu L, Zamani AA. Clinical and neuroradiographic manifestations of eastern equine encephalitis. N Engl J Med 1997;336:1867-74.

22. Babi MA, Raleigh T, Shapiro RE, McSherry J, Applebee A. MRI and encephalography in fatal eastern equine encephalitis. Neurology 2014;83:1483.

23. Nickerson JP, Kannabiran S, Burbank HN. MRI findings in eastern equine encephalitis: the "parenthesis" sign. Clin Imaging 2016;40:222-3.

24. World Health Organization. Global tuberculosis report 2019. Available online: https://www.who.int/tb/ publications/global_report/en/. (Accessed on October 28, 2019).

25. CDC - National Center for Health Statistics Tuberculosis Data and Statistics. Available online: https:// www.cdc.gov/tb/statistics/default.htm. August 10, 2019.

26. Langer AJ, Navin TR, Winston CA, LoBue P. Epidemiology of Tuberculosis in the United States. Clin 
Chest Med 2019;40:693-702.

27. Méchaï F, Bouchaud O. Tuberculous meningitis: Challenges in diagnosis and management. Rev Neurol (Paris) 2019;175:451-7.

28. Chin JH. Tuberculous meningitis: Diagnostic and therapeutic challenges. Neurol Clin Pract 2014;4:199-205.

29. Weingarten K, Zimmerman RD, Becker RD, Heier LA, Haimes AB, Deck MD. Subdural and epidural empyemas: MR imaging. AJR Am J Roentgenol 1989;152:615-21.

30. McIntyre PB, Lavercombe PS, Kemp RJ, McCormack JG. Subdural and epidural empyema: diagnostic and therapeutic problems. Med J Australia 1991;154:653-7.

31. Lundy P, Kaufman C, Garcia D, Partington MD, Grabb PA. Intracranial subdural empyemas and epidural abscesses in children. J Neurosurg Pediatr 2019;24:14-21.

32. Mattogno PP, La Rocca G, Signorelli F, Visocchi M. Intracranial subdural empyema: diagnosis and treatment update. J Neurosurg Sci 2019;63:101-2.

33. Bartt RE. Cranial epidural abscess and subdural empyema. Handb Clin Neurol 2010;96:75-89.

34. Tsuchiya K, Osawa A, Katase S, Fujikawa A, Hachiya J, Aoki S. Diffusion-weighted MRI of subdural and epidural empyemas. Neuroradiology 2003;45:220-3.

35. Haimes AB, Zimmerman RD, Morgello S, Weingarten $\mathrm{K}$, Becker RD, Jennis R, Deck MD. MR imaging of brain abscesses. AJR Am J Roentgenol 1989;152:1073-85.

36. Shih RY, Koeller KK. Bacterial, Fungal, and Parasitic Infections of the Central Nervous System: RadiologicPathologic Correlation and Historical Perspectives. Radiographics 2015;35:1141-69.

37. Rath TJ, Hughes M, Arabi M, Shah GV. Imaging of cerebritis, encephalitis, and brain abscess. Neuroimaging Clin N Am 2012;22:585-607.

38. Ganau M, Mankad K, Srirambhatla UR, Tahir Z, D'Arco F. Ring-enhancing lesions in neonatal meningitis: an analysis of neuroradiology pitfalls through exemplificative cases and a review of the literature. Quant Imaging Med Surg 2018;8:333-41.

39. Lai PH, Chung HW, Chang HC, Fu JH, Wang PC, Hsu SH, Hsu SS, Lin HS, Chuang TC. Susceptibility-weighted imaging provides complementary value to diffusionweighted imaging in the differentiation between pyogenic brain abscesses, necrotic glioblastomas, and necrotic metastatic brain tumors. Eur J Radiol 2019;117:56-61.

40. Fukui MB, Williams RL, Mudigonda S. CT and MR imaging features of pyogenic ventriculitis. AJNR Am J Neuroradiol 2001;22:1510-6.

41. Pezzullo JA, Tung GA, Mudigonda S, Rogg JM. Diffusion- weighted MR imaging of pyogenic ventriculitis. AJR Am J Roentgenol 2003;180:71-5.

42. García-Cabrera E, Fernández-Hidalgo N, Almirante B, Ivanova-Georgieva R, Noureddine M, Plata A, Lomas JM, Gálvez-Acebal J, Hidalgo-Tenorio C, RuízMorales J, Martínez-Marcos FJ, Reguera JM, de la TorreLima J, de Alarcón González A; Group for the Study of Cardiovascular Infections of the Andalusian Society of Infectious Diseases; Spanish Network for Research in Infectious Diseases. Neurological complications of infective endocarditis: risk factors, outcome, and impact of cardiac surgery: a multicenter observational study. Circulation 2013;127:2272-84.

43. Okazaki S, Yoshioka D, Sakaguchi M, Sawa Y, Mochizuki $\mathrm{H}$, Kitagawa $\mathrm{K}$. Acute ischemic brain lesions in infective endocarditis: incidence, related factors, and postoperative outcome. Cerebrovasc Dis 2013;35:155-62.

44. Hess A, Klein I, Iung B, Lavallée P, Ilic-Habensus E, Dornic Q, Arnoult F, Mimoun L, Wolff M, Duval $\mathrm{X}$, Laissy JP. Brain MRI findings in neurologically asymptomatic patients with infective endocarditis. AJNR Am J Neuroradiol 2013;34:1579-84.

45. Shen G, Shen X, Pu W, Zhang G, Lerner A, Gao B. Imaging of cerebrovascular complications of infection. Quant Imaging Med Surg 2018;8:1039-51.

46. Faisal W, Burnton G, Imlay-Gillespie L, Robilliard J. Cerebral abscesses and septic pulmonary emboli due to Serratia marcescens infection arising from a Portacath. J Microbiol Immunol Infect 2010;43:538-41.

47. Antinori A, Larussa D, Cingolani A, Lorenzini P, Bossolasco S, Finazzi MG, Bongiovanni M, Guaraldi G, Grisetti S, Vigo B, Gigli B, Mariano A, Dalle Nogare ER, De Marco M, Moretti F, Corsi P, Abrescia N, Rellecati P, Castagna A, Mussini C, Ammassari A, Cinque P, d'Arminio Monforte A; Italian Registry Investigative NeuroAIDS. Prevalence, associated factors, and prognostic determinants of AIDS-related toxoplasmic encephalitis in the era of advanced highly active antiretroviral therapy. Clin Infect Dis 2004;39:1681-91.

48. Matinella A, Lanzafame M, Bonometti MA, Gajofatto A, Concia E, Vento S, Monaco S, Ferrari S. Neurological complications of HIV infection in pre-HAART and HAART era: a retrospective study. J Neurol 2015;262:1317-27.

49. Bowen LN, Smith B, Reich D, Quezado M, Nath A. HIVassociated opportunistic CNS infections: pathophysiology, diagnosis and treatment. Nat Rev Neurol 2016;12:662-74.

50. Lee GT, Antelo F, Mlikotic AA. Cerebral Toxoplasmosis. 
RadioGraphics 2009;29:1200-5.

51. Garg RK, Sinha MK. Multiple Ring-Enhancing Lesions of the Brain. J Postgrad Med 2010;56:307-16.

52. Chong-Han CH, Cortez SC, Tung GA. DiffusionWeighted MRI of Cerebral Toxoplasmosis Abscess. AJR 2003;181:1711-4.

53. Williamson PR, Jarvis JN, Panackal AA, Fisher MC, Molloy SF, Loyse A, Harrison TS. Cryptococcal meningitis: epidemiology, immunology, diagnosis and therapy. Nat Rev Neurol 2017;13:13-24.

54. Spec A, Powderly WG. Cryptococcal meningitis in AIDS. Handb Clin Neurol 2018;152:139-50.

55. Lee SC, Dickson DW, Casadevall A. Pathology of cryptococcal meningoencephalitis: analysis of 27 patients with pathogenetic implications. Hum Pathol 1996;27:839-47.

56. Kashef Hamadani BH, Franco-Paredes C, McCollister B, Shapiro L, Beckham JD, Henao-Martínez AF. Cryptococcosis and cryptococcal meningitis: New predictors and clinical outcomes at a United States academic medical centre. Mycoses 2018;61:314-20.

57. Zhai S, Brew BJ. Progressive multifocal leukoencephalopathy. Handb Clin Neurol 2018;152:123-37.

58. Kartau M, Sipilä JO, Auvinen E, Palomäki M, Verkkoniemi-Ahola A. Progressive Multifocal Leukoencephalopathy: Current Insights. Degener Neurol Neuromuscul Dis 2019;9:109-21.

59. Berger JR, Aksamit AJ, Clifford DB, Davis L, Koralnik IJ, Sejvar JJ, Bartt R, Major EO, Nath A. PML diagnostic criteria: consensus statement from the AAN Neuroinfectious Disease Section. Neurology 2013;80:1430-8.

60. Mornese Pinna S, Scarvaglieri E, Milia MG, Imperiale D, Ghisetti V, Audagnotto S, Prochet A, Lipani F, Bonora S, Di Perri G, Calcagno A. Detectable cerebrospinal fluid JCV DNA in late-presenting HIV-positive patients: beyond progressive multifocal leukoencephalopathy? J Neurovirol 2017;23:763-7.

61. Küker W, Mader I, Nägele T, Uhl M, Adolph C, Klose U, Herrlinger U. Progressive multifocal leukoencephalopathy: value of diffusion-weighted and contrast-enhanced magnetic resonance imaging for diagnosis and treatment control. Eur J Neurol 2006;13:819-26.

62. Sahraian MA, Radue EW, Eshaghi A, Besliu S, Minagar A. Progressive multifocal leukoencephalopathy: a review of the neuroimaging features and differential diagnosis. Eur J Neurol 2012;19:1060-9.
63. Berger JR, Levy RM, Flomenhoft D, Dobbs M. Predictive factors for prolonged survival in acquired immunodeficiency syndrome-associated progressive multifocal leukoencephalopathy. Ann Neurol 1998;44:341-9.

64. Krey L, Raab P, Sherzay R, Berding G, Stoll M, Stangel M, Wegner F. Severe Progressive Multifocal Leukoencephalopathy (PML) and Spontaneous Immune Reconstitution Inflammatory Syndrome (IRIS) in an Immunocompetent Patient. Front Immunol 2019;10:1188.

65. Schweitzer AD, Parikh NS, Askin G, Nemade A, Lyo J, Karimi S, Knobel A, Navi BB, Young RJ, Gupta A. Imaging characteristics associated with clinical outcomes in posterior reversible encephalopathy syndrome. Neuroradiology 2017;59:379-86.

66. Issa K, Diebo BG, Faloon M, Naziri Q, Pourtaheri S, Paulino CB, Emami A. The Epidemiology of Vertebral Osteomyelitis in the United States From 1998 to 2013. Clin Spine Surg 2018;31:E102-8.

67. Allison DC, Holtom PD, Patzakis MJ, Zalavras CG. Microbiology of bone and joint infections in injecting drug abusers. Clin Orthop Relat Res 2010;468:2107-12.

68. Kim UJ, Bae JY, Kim SE, Kim CJ, Kang SJ, Jang HC, Jung SI, Song KH, Kim ES, Kim HB, Park WB, Kim NJ, Park KH. Comparison of pyogenic postoperative and native vertebral osteomyelitis. Spine J 2019;19:880-7.

69. Aagaard T, Roed C, Dragsted C, Skinhøj P. Microbiological and therapeutic challenges in infectious spondylodiscitis: a cohort study of 100 cases, 2006-2011. Scand J Infect Dis 2013;45:417-24.

70. Berbari EF, Kanj SS, Kowalski TJ, Darouiche RO, Widmer AF, Schmitt SK, Hendershot EF, Holtom PD, Huddleston PM 3rd, Petermann GW, Osmon DR, Infectious Diseases Society of America. 2015 Infectious Diseases Society of America (IDSA) Clinical Practice Guidelines for the Diagnosis and Treatment of Native Vertebral Osteomyelitis in Adults. Clin Infect Dis 2015;61:e26-46.

71. Zimmerli W. Clinical practice. Vertebral osteomyelitis. N Engl J Med 2010;362:1022-9.

72. McHenry MC, Easley KA, Locker GA. Vertebral osteomyelitis: long-term outcome for 253 patients from 7 Cleveland-area hospitals. Clin Infect Dis 2002;34:1342-50.

73. Numaguchi Y, Rigamonti D, Rothman MI, Sato S, Mihara F, Sadato N. Spinal Epidural Abscess: Evaluation with Gadolinium-Enhanced MR Imaging. Radiographics 1993;13:545-59.

74. Darouiche RO, Hamill RJ, Greenberg SB, Weathers SW, 
Musher DM. Bacterial spinal epidural abscess. Review of 43 cases and literature survey. Medicine (Baltimore) 1992;71:369-85.

75. Eastwood JD, Vollmer RT, Provenzale JM. Diffusionweighted imaging in a patient with vertebral and epidural abscesses. AJNR Am J Neuroradiol 2002;23:496-8.

Cite this article as: Li S, Nguyen IP, Urbanczyk K. Common infectious diseases of the central nervous system-clinical features and imaging characteristics. Quant Imaging Med Surg 2020;10(12):2227-2259. doi: 10.21037/qims-20-886
76. Shah LM, Salzman KL. Imaging of spinal metastatic disease. Int J Surg Oncol 2011;2011:769753.

77. Holtås S, Heiling M, Lönntoft. Spontaneous Spinal Epidural Hematoma: Findings at MR Imaging and Clinical Correlation. Radiology 1996;199:409-13. 